\title{
La dottrina della circolazione del sangue e la Scuola Galileiana 1636-61
}

Dall'Istituto di Storia della Medicina, Università degli Studi, Milano

\section{Luigi Belloni}

Sommario: 1. I Barberini e B. Castelli, capo dei Galileiani di Roma. W. Harvey a Roma e a Napoli nel 1636: suoi contatti col Card.F. Barberini e con M. A. Severino. - 2. Interesse di R. Magiotti nel 1637 per la circolazione del sangue e per le anatomie del «medico todesco». 3. P.M. Schlegel a Roma nel 1636/7. - 4. G. Trulli, chirurgo «a' servizi del S. Cardl Barberino». - 5. G. Trulli e la dottrina della circolazione: testimonianze nel suo epistolario col Severino e in opere del Severino. - 6. G. A. Borelli e M. Malpighi: iatromeccanica e scoperte strutturistiche (miocardio, capillari sanguigni).

1. I Barberini e B. Castelli, capo dei Galileiani di Roma. W. Harvey a Roma e a Napoli nel 1636: suoi contatti col Card.F. Barberini e con M.A.Severino. Siamo negli anni in cui il tragico destino del fondatore della scienza moderna va inesorabilmente avviandosi alla conclusione. Il 2 gennaio 1638, «fatto irreparabilmente da un mese in qua del tutto cieco », il confinato di Arcetri annuncia con disperazione: «quel cielo, quel mondo e quello universo che io con mie meravigliose osservazioni e chiare dimostrazioni avevo ampliato per cento e mille volte più del comunemente veduto da' sapienti di tutti i secoli passati, ora per me s'è sì diminuito e ristretto, ch'e' non è maggiore di quel che occupa la persona mia ». ${ }^{1}$

Dopo l'infausto processo del 1633, lo spirito scientifico di Galileo Galilei (1564-1642) è mantenuto in vita, nella Roma di Urbano VIII, dagli studiosi raccolti attorno a B. Castelli. Spiccano, fra essi, i nomi di G.A.Borelli e di E.Torricelli. Quest'ultimo integra, con R.Magiotti e A.Nardi, il celebre «triumvirato» che ricorre nel carteggio Galileiano. Altro nome interessante per il nostro lavoro è quello di P.B.Borghi.

Benedetto Castelli ${ }^{2}$ (1578-1643) era lettore di matematica all'Università di Roma (detta anche Archiginnasio Romano o «Sapienza»). Questa carica gli era stata affidata da Urbano VIII (Maffeo Barberini, 1568-1644), che, poco dopo la sua elevazione al soglio pontificio (1623), lo aveva chiamato dall'Università di Pisa per affidargli l'istruzione del nipote Taddeo (1604-47).

1 Le opere di Galileo Galilei (ediz. nazion.) 17, 247.

2 G.L.Masetti Zannini, La vita di Benedetto Castelli, Brescia 1961. 
A quest'ultimo sono, per l'appunto, dedicate le Dimostrazioni geometriche della misura dell'acque correnti (Roma 1628) con cui il Castelli «elevò lo studio dell'idraulica a dignità di scienza ${ }^{3}{ }^{3}$

Meglio noto alla storia della scienza è Francesco Barberini (1597-1679), fratello maggiore di Taddeo, che lo zio pontefice creò cardinale il 2 ottobre 1623. Due giorni prima, l'Accademia dei Lincei ${ }^{4}$, l'illustre consesso scientifico che vantava il nome del Galilei, lo aveva eletto suo membro. Nel 1630 il collega Linceo Francesco Stelluti (1577-1646) gli dedicò il Persio tradotto con la nuova versione della Melissographia Lincea (1625) in esso contenuta, grazie alla quale le api araldiche della famiglia Barberini possono vantare il primato della inconografia biomicroscopica ${ }^{5}$. «Protettore di letterati, artisti e scienziati», il cardinal Francesco Barberini «si compiaceva di ospitare nella sua Corte eletti ingegni». ${ }^{6}$ Benedetto Castelli apparteneva, per l'appunto, alla sua corte $^{7}$; e il Linceo Cassiano dal Pozzo (1583-1657) ${ }^{8}$ ricopriva la carica di maestro di casa.

Nella parte indipendente del viaggio sul continente al fianco dell'Arundel ${ }^{9}$, William Harvey (1578-1657) soggiornò a Roma nei mesi di settembre-ottobre del 1636: e in tale occasione egli ricevette cortesie e favori ${ }^{10}$ dal cardinale «Protector Angliae», ${ }^{11}$ che era per l'appunto Francesco Barberini.

Da Roma, l'Harvey raggiunse Napoli ${ }^{12}$, dove si incontrò con Marco Aurelio Severino $(1580-1656)^{13}$, come apprendiamo da lettere dell'Harvey al

3 Così afferma A.Favaro nel breve profilo del Castelli in Le opere di G. G. 20, 412.

4 Svariate notizie su Francesco Barberini sono infatti reperibili in G. GabrIELI, Il carteggio Linceo della vecchia Accademia di Federico Cesi (1603-1630), Roma 1938-1942.

5 L. Belloni, Il primo ventennio della microscopia (Galilei 1610 - Harvey 1628): dalla microscopia alla anatomia microscopica dell'insetto, Clio Medica, 4, (1969) 179-190.

${ }^{6}$ P. Pecchial, I Barberini, Roma 1959, 157.

7 Pecchiai, 158.

${ }^{8}$ G.Lumbroso, Notizie sulla vita di Cassiano dal Pozzo, Miscellanea di Storia Italiana edita per cura della Regia Deputazione di Storia Patria 15 (1874) 129-388.

${ }^{9}$ Per il tracciato geografico di questo viaggio cfr. K. D. Keele, William Harvey. The man, the physician, and the scientist, London/Edinburgh 1965, 40; G. KEYNES, The Life of William Harvey, Oxford 1966, 231.

10 KeYNES, The Life of W.H., 258 e 261-262.

11 J. Wodka, Zur Geschichte der nationalen Protektorate der Kardinäle an der römischen Kurie (= Band IV der «Publikationen des ehemaligen Österreichischen Historischen Instituts in Rom »), Innsbruck/Leipzig 1938, 14 (nota 13) e 119.

12 Keynes, The Life of W.H., 258-259.

${ }^{13}$ L.Belloni, Severinus als Vorläufer Malpighis, Beiträge zur Geschichte der Medizin und der Naturwissenschaften, Festschrift für Professor Dr. Rudolph Zaunick zum 70. Geburtstag am 26. August 1963 (= Nova Acta Leopoldina 27, N.167), 213-224 (1963). 
Severino ${ }^{14}$ e del Severino all'Harvey ${ }^{15}$ conservate alla Biblioteca Lancisiana $^{16}$ in Roma e segnalate da Virgilio Ducceschi (1871-1952) ${ }^{17}$.

\section{Interesse di R. Magiotti nel 1637 per la circolazione del sangue e per le} anatomie del «medico todesco». - Pochi mesi dopo il soggiorno di Harvey, è documentabile un vivo interesse per la circolazione del sangue in un Galileiano di Roma. Esso è manifestato il 25 aprile 1637 da Raffaello Magiotti (1597-1658) in una lettera ${ }^{18}$ a Famiano Michelini (1604-65) ${ }^{19}$, uno dei compagni di Galileo in Firenze e, più tardi (1648-55), lettore di matematica a Pisa. L'uno e l'altro, mittente e destinatario, sono, al pari del Castelli, particolarmente interessati all'idraulica. Quantunque stesa, come riconosce le stesso Magiotti, «con modo pur assai confuso e con mente astrattissima $»,{ }^{20}$ la sua descrizione è tuttavia meritevole di venire integralmente riportata:

Confesso non haver, in questo punto, spirito di trattar delli spiriti vitali; pur tuttavia, per non la lasciar affatto a bocca aperta, gliene darò un poco di saggio così al barlume, non

14 Lettera dell'Harvey al Severino, non datata: «De singulari erga me effusa humanitate, et benevolentia tua, Marce Aureli Severine doctissime, cum anno praeterito Neapolim peregrinando inviserem, gratias (nunc), quas possim, ago maximas», ms. Lancis.49, c. $92^{\mathrm{v}}$; ms. Lancis. 54, c. $61^{\mathrm{r}}\left(=66^{\mathrm{r}}\right)$.

15 Lettera del Severino all'Harvey, da Napoli $1^{\circ}$ marzo 1638: «... gaudeo item mihi de mea bona fortuna, qui de facie te novum Democritum, Herophilumque novam cognoverim: notitiae vero meae fructus quotidie suavissimos, optatissimosque tua singulari benignitate percipiam », ms. Lancis. 68, c. $186(=189)$.

Lettera del Severino all'Harvey, da Napoli $1^{\circ}$ aprile 1639: «Postquam tua nobis illuxit claritas semel, novimusque maiorem, quam fama praedixerat, tuam de facie dignitatem: ab illo non tuus est unquam lapsus de pectore vultus, neque abscessit a nobis immensum colendi, venerandique tui desiderium", ms. Lancis. 68, c. 187 (=190).

16 I rapporti del Severino con l'Harvey e la sua posizione di fronte alla dottrina della circolazione saranno oggetto di altro lavoro, largamente basato anche sul materiale manoscritto della Biblioteca Lancisiana, e non soltanto sull'epistolario del Severino (con W. Harvey, G. Ent, J. Houghton, etc.), ma anche sul volume da lui dedicato all'argomento (ms. Lancis. 34).

17 V.Ducceschi, L'epistolario di Marco Aurelio Severino (1580-1656). Un fondo per la storia della medicina nella prima metà del secolo XVII., Rivista di storia delle scienze mediche e naturali 14, (1923) 213-223.

18 Le opere di G.G. 17, 64-66.

19 G. Giovannozzi, Scolopi Galileiani, Atti della Società Colombaria di Firenze degli anni

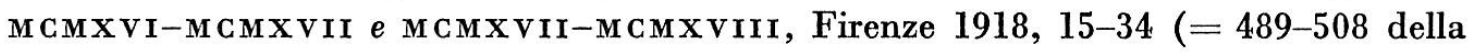
numerazione globale iniziata con l'anno accademico 1910/11).

20 Cfr. la nota 28. 
potendo noi per adesso haver commodità di veder insieme anatomie. Sono molti anni che un medico milanese osservò negli animali pasciuti di fresco e poi ammazzati (massime nei cani), che nel mesentereo sono molte vene lattee ${ }^{21}$, quali da tutti gl'intestini tirano succo overo chilo alla volta del pancreas, e per quello al fegato ${ }^{22}$ et alla vena cava, per la quale finalmente s'annida, si riscalda e concuoce dentro al destro ventricolo del cuore; di quivi dalla vena arteriosa passa a refrigerarsi nel polmone per meglio conquocersi, e dal polmone per l'arteria venosa torna nel sinistro ventricolo del cuore, dove si fa l'ultima concotione. Di là per l'arteria magna, e da lei per tutte l'arterie, si sparge il sangue spiritoso per tutto il corpo. Così si diffondono gli spiriti et il calore, e così il moto del pulsare, a tutte le membra. Dalle membra tutte succhiano le vene capillari il sangue, quale era stato portato dalle arterie per nutrir le parti, come se fussero tante radiche e barbe; e riconducano il sangue così con pochissimi spiriti al quore per la vena porta ${ }^{23}$, acciò là di nuovo con qualche portione di nuovo chilo per opera delle vene lattee si riscaldi e conquoca.

Questa è la circulatione che fa il sangue in noi, osservata alli tempi nostri, e bastante a rivolger tutta la medicina ${ }^{24}$, sì come l'inventione del telescopio ha rivolta tutta l'astronomia, la bossola l'economia, e l'artiglieria tutta l'arte militare.

Queste vene lattee non sono vene mesaraiche, anzi non sono visibili se l'animale fusse estenuato e non pasciuto poco avanti la morte: però nell'huomo si vedono di rado. Argomento certo che l'arterie portino dal centro alla circonferenza, è che per l'arterie si trovano molte valvule, overo animelle, che lasciano bene passare il sangue dal cuore alle membra, ma non rientrare; e per il contrario nelle vene l'animelle lasciano da ogni banda tornare il sangue al core, ma non uscire. Ma de valvulis è fuora un libro ${ }^{25}$. L'arterie sono più carnee che le vene, perchè devono rattenere gli spiriti con il sangue, dove le vene non portano se non il sangue; e questa loro carnosità sempre più scema, quanto più si slontana dal cuore, perchè sempre manco spiriti deveno rattenere. Nè importa che le vene sieno sì grosse $\mathrm{e}$ l'arterie sì sottili, perchè il sangue spiritoso presto passa per loro.

S'io havessi meglio distesa questa nuovità, haverei havuto a caro che l'havessi intesa il Sig. G. G. S'ella si rincuora di meglio raccontargliene, facc'ella. Vero è ch'io ho havuto grandissimo gusto questo anno in alcune anatomie fatte da un medico todesco ${ }^{26}$, persona di rara et esquisita curiosità in buona filosophia e medicina.

${ }^{21} \mathrm{La}$ scoperta delle vene lattee, o vasi chiliferi, fu compiuta a Milano il 23 luglio 1622 da Gaspare Aselli (1581 ca.-1625), nativo di Cremona. La descrizione della scoperta trovasi nella sua opera postuma De lactibus, sive lacteis venis, quarto vasorum meseraicorum genere novo invento (Milano 1627). Cfr. L. BELloni, La medicina a Milano fino al Seicento, Storia di Milano della Fondazione Treccani degli Alfieri 11 (1958) 678-682.

22 Scoperti i vasi chiliferi, l'Aselli aveva continuato a ritenere, con Galeno, che il chilo, assorbito dall'intestino, avesse per meta il fegato. Il dotto toracico fu scoperto nel 1647 da Jean Pecquet (1622-74).

${ }^{23} \mathrm{O}$ meglio, per la vena cava.

${ }^{24} \mathrm{Il}$ corsivo è nostro!

25 Allude verosimilmente al De venarum ostiolis (Venezia 1603) di Gerolamo Fabrizi da Acquapendente (1533 ca.-1619). Cfr. G.FABrizi da Acquapendente, Delle valvole delle vene (De venarum ostiolis). Ristampa, traduzione e introduzione a cura di Felice Grondona, Milano 1966. 
Si tratta verosimilmente dello stesso «medico todesco » citato dal Magiot$\mathrm{ti}^{27}$ il 21 marzo 1637 in una lettera a Galileo.

Galileo lesse «con qualche gusto la circolatione del sangue» ${ }^{28}$ descritta dal Magiotti al Michelini: e quest'ultimo, partendo da elementi affini a quelli esposti dal Magiotti, andò man mano costruendo e perfezionando un suo sistema medico-igienico ${ }^{29}$, che intendo approfondire in altra sede, limitandomi per ora a metterne in evidenza i legami con la dottrina della circolazione del sangue e con la medicina statica di Santorio Santorio (1561-1636).

Dopo un decennio circa, il Magiotti ritornò, sia pure incidentalmente, sulla circolazione del sangue: e ciò fece nell'opuscolo Renitenza certissima dell'acqua alla compressione dichiarata con varij scherzi in occasione d'altri problemi curiosi (Roma 1648) in cui descrisse e raffigurò il dispositivo fisico che assumerà più tardi il nome di «ludione Cartesiano»o «diavoletto di Descartes ${ }^{30}$ I progressi della scienza - afferma il Magiotti - derivano dal nostro lavoro di ricerca, e non perchè «la natura, per non se ne stare in otio, produce qualche cosetta di nuovo »: infatti, «le valvule delle nostre vene e dell'arterie, la circolation del sangue ${ }^{31}$, la palpitation del cuore, sono antiche quanto l'istesso Adamo: e la speculatione è tanto nuova, quanto l'Herveio, ingegno sottilissimo del nostro secolo».

26 Il corsivo è nostro!

27 «... qua si trova un medico todesco, anatomista raro, quale mostra in fatto assaissimi errori de natura animalium; e quand'io gli contai del cavallo di Gattamelata, che sta sopra dua gambe dalla medesima banda, contro il detto d'Aristotile, rise veramente di tutto core; et ogni giorno porta qualche luogo, per farci sempre più ridere ", Le opere di G.G. 17, 50-51.

${ }^{28}$ Come ci fa sapere lo stesso Magiotti in una lettera a Galileo del 16 maggio 1637: «Non è poco che V.S. molto Illre et Ecc ${ }^{\mathrm{ma}}{ }^{\mathrm{r}}$ habbia letto con qualche gusto la circolatione del sangue, scritta da me con modo pur assai confuso e con mente astrattissima, sebene io non l'amo più per giudice delle parole che delle cose », Le opere di G. G. 17, 80.

29 G.Targioni-Tozzetti, Atti e memorie inedite dell'Accademia del Cimento e notizie aneddote dei progressi delle scienze in Toscana, Firenze 1780, 2/1, 221-234 e 3, 322-345.

Discorsi di D.Famiano Michelini sopra la Sanità, Biblioteca Naz. Marciana, Venezia, mss. Italiani, classe 3, numero 19, collocazione 5006; Biblioteca Nazionale di Firenze, ms. Galil. 127, cc. 86-107.

30 L. Belloni, Schemi e modelli della macchina vivente nel Seicento. Con ristampa della lettera di R.Magiotti 'Renitenza certissima dell'acqua alla compressione' (il «diavoletto di Descartes»), Physis 5, (1963) 259-298 (cfr. 272).

31 Il corsivo è nostro! 
Antonio Nardi ${ }^{32}$, che col Magiotti e il Torricelli integrava, come si è detto, il «triumvirato » Galileiano, ha lasciato un grosso manoscritto quasi interamente inedito - le Scene - che egli portò verosimilmente a termine entro il 1645 , anno in cui sembrava volesse darlo alle stampe ${ }^{33}$. Su indicazione di Raffaello Caverni (1837-1900) ${ }^{34}$, mette conto di stralciare un brano di questo manoscritto ${ }^{35}$ relativo alla circolazione del sangue:

... l'orecchie del Cuore sono una natura di mezzo, et un certo legame tra il Cuore et i vasi venali et arteriali: anche sono le prime, e l'ultime a vivere, e moversi tra le parti solide dell'animale. Battono non in virtù propria, ma del sangue spiritoso, il quale come fuoco artifiziosissimo ha movimento ed atto perpetuo, insino che resta sangue. Al battere delle orecchie segue il restringersi, e allargarsi del Cuore, poichè riempito di sangue il ventricello destro dalla vena cava, e dalla destra orecchia, restringesi per il soverchio caldo, e discaccia il sangue per i vasi, e di nuovo ritornando al primiero, e naturale stato, torna a riempirsi alternam. ${ }^{\text {te }}$, e così un certo moto circolare, e perpetuo formasi del sangue, mentre dal destro ventricello se ne passa per i condotti al polmone, e quindi se ne ritorna al sinistro, a che ancora il moto del polmone serve: ed osservisi, che il cuore non solo ha il movimento sudetto di restringersi, et allargarsi, ma anche l'arterie, massime maggiori, ed anche la vena cava presso il Cuore, e questo moto seconda per consenso quello del Cuore: quindi ancora il sangue per le vene passa dalle parti al cuore, e per l'arterie dal Cuore passa alle parti, e l'uno spinge l'altro; e non è dubbio, che questa moderna osservazione del moto circolare del sangue non sia una delle belle cose, che si sia mai trovata nell'arte ${ }^{36}$; onde moltissime considerazioni farsi potrebbono, di che vedasi l'Harveio; è ben vero, che molto paradossa parrà l'opinione di questo dottissimo huomo, mentre nell'inferior ventre pensasi, che le vespe, ed altri somiglianti animali habbiano il Cuore ${ }^{37}$; perchè se dal battere una sua parte ciò si potesse argomentare, seguiriane, che gli animali più perfetti l'havessino in capo, vedendosi il cervello battere. Alcuno più tosto penserà che la parte battente nell'inferior ventre delle vespe siano i vasi seminali; nulla non di meno affermo in materia così dubbia per che sperienzia fatto non ne ho, nè meno rifiuto il parere di tal autore, quale concorda col mio, cioè, che gli insetti spirino per le fascie.

3. P.M.Schlegel a Roma nel 1636/7. - Nel 1635 Emilio Parisano (1567 a 1643), medico Veneto, pubblicò una serie di «refutationes» alla dottrina

32 Da non confondere con Giovanni Nardi (1585-1654), medico del granduca Ferdinando II di Toscana, che è ben noto alla letteratura Harveyana.

33 Dal breve profilo biografico in Le opere di G. G. 20, 492.

34 R. CAVERNI, Storia del metodo sperimentale in Italia, Firenze 1893, 3, 155.

35 Biblioteca Nazionale di Firenze, ms. Galil. 130, 1096-1098.

36 Il corsivo è nostro!

${ }^{37}$ Si veda l'articolo di Belloni citato alla nota 5. Le stesse osservazioni microscopiche dell'Harvey sul vaso dorsale pulsante nell'addome degli insetti sono ricordate anche dal Berigardus (Claude Guillermet de Bérigard; 1592 ca.-1663) nel Circulus Pisanus De veteri et peripatetica philosophia In tres libros Aristotelis de anima, Udine 1643, 28-29. 
circolatoria, intercalandole ai relativi brani dell'opera dell'Harvey, che risultò pertanto, anche se non del tutto integralmente, ristampata: questa, infatti, apparsa a Venezia nel 1635, è considerata ${ }^{38}$ come la prima ristampa del De motu cordis et sanguinis (1628) dell'Harvey. A controbattere le «refutationes» del Parisano si leverà l'Apologia pro circulatione sanguinis (Londra 1641) di George Ent (1604-89): lo stesso Ent che, laureandosi ${ }^{39}$ a Padova il 28 aprile 1636, ricevette in dedica dal danese Johannes Rhode (ca. 1587-1659) una composizione poetica con riferimento all'Harvey e alla circolazione. Quei versi sembravano presagire il non lontano incontro fra l'Ent e l'Harvey, che avvenne a Roma il 5 ottobre 1636, e l'amicizia che in questa occasione fu stretta fra i due medici inglesi ${ }^{40}$.

Il 22 marzo 1636 - poco più di un mese prima dell'Ent - si era laureato a Padova ${ }^{41}$ il tedesco Paul Marquard Schlegel (Sleghelius, 1605-53) ${ }^{42}$, che pubblicherà la De sanguinis motu commentatio (Amburgo 1650) ${ }^{43}$ quando ormai da un ventennio si era fatto paladino della dottrina circolatoria e l'aveva dimostrata con pubbliche dissezioni anche nei paesi stranieri da lui frequentati nel periodo 1631-8:

Incredibile vero est dictu, quanta jam sit hujus doctrinae apud me firmitas, et certitudo, cum nempe ea viginti annis animo agitata, et multiplici enchiresi a me examinata, nulla sui parte erronea deprehensa: et, jam iterum sub incudem revocata, adeo invictam se praebuerit ... In peregrinatione itidem mea per Belgium, Angliam, Galliam, Italiam, Germaniam, cum doctissimis quibusq. Medicis, et Anatomicis collocutus, non memini ullius, qui contra aliquid moliri potuerit, licet in publicis dissectionibus apud exteros saepius sit a me proposi$t a^{44}$.

38 G. Keynes, A Bibliography of the Writings of DR William Harvey, Cambridge 1953, 8.

${ }^{39}$ British Museum 551.6.1. (3). En t (Sir George): Laureae Apollinari. Praes. B.Sylvatico ... promotore ... D. Sala ... G. Ent in ... Lyceo Patavino 28. Aprilis, 1636. collatae amicorum applausus. Patavii [1636], $4^{\circ}$. La citazione è tratta da British Museum General Catalogue of Printed Books, 67 (ediz. 1955) 334.

40 Keynes, The Life of W.H., 257-259.

41 F. Weigle, Die deutschen Doktorpromotionen in Philosophie und Medizin an der Universität Padua von 1616-1663, Quellen und Forschungen aus italienischen Archiven und Bibliotheken 45 (1965) 325-384 (cfr. p. 345, n. 250).

Acta Nationis Germanicae Artistarum 1616-1636 a cura di Lucia Rossetti, Padova 1967, 404.

42 Naturforschung und Naturlehre im alten Hamburg, Hamburg 1928, 23-25.

43 K. E. Rотнsснuн, Jean Riolan jun. (1580-1657) im Streit mit Paul Marquart Schlegel (1605-1653) um die Blutbewegungslehre Harveys, Gesnerus 21 (1964) 72-82.

44 Il corsivo è nostro! Questo brano autobiografico dello Schlegel è tratto dalla prefazione alla sua De sanguinis motu commentatio (Amburgo 1650. 
Reduce dai cimenti superati a Parigi (1632) e a Montpellier (1634), lo Schlegel si immatricolò ${ }^{45}$ all'Università di Padova il 20 ottobre 1635: e in seno a questa cercò di aggiungere al serto delle proprie vittorie anche la conversione di Johann Vesling (1598-1649) ${ }^{46}$, che, inizialmente contrario, «pedetentim tamen se induci passus est, non parum huc faciente frequentiore nostra consuetudine, ut me ex Italia discedente Harvei partes omnino susceperit ».47 Nel 1638 lo Schlegel rientrò in Germania per ricoprire la cattedra di anatomia, chirurgia e botanica di Jena; e in quello stesso anno fu ospite, per 4 mesi, di Kaspar Hofmann (1572-1648) in Altdorf, senza però riuscire a convertirlo ${ }^{48}$.

Laureato, come si è detto, a Padova il 22 marzo 1636, lo Schlegel non tardò a prendere le disposizioni per trasferirsi a Roma ${ }^{49}$, dove la sua presenza è assicurata da svariati documenti epistolari. Uno di essi appartiene al gruppo di lettere allo Schlegel indicatomi da Luigi Amabile (1828-92) ${ }^{50}$ : ed è, precisamente, la lettera ${ }^{51}$ datata Messina 29 settembre 1636 e inviata allo Schlegel a Roma dal romano Pietro Castelli ${ }^{52}$, professore di medicina a Messina. Il discorso, in essa, tocca anche la circolazione del sangue, argomento sul quale il Castelli era stato colto evidentemente impreparato dallo Schlegel:

... quid autem de circulatione sanguinis scripserit Harvejus me latet; nec dum enim ejus liber, nec opinio ad me pervenit. Unde quam circulationem intelligat, non percipio. Certum quidem habeo ex Hippocrate ${ }^{53}$ dari circulum et fluxum sanguinis, qui cum nunquam quies-

45 Cfr. nota 41.

46 Sulla posizione del Vesling in merito alla dottrina della circolazione sono da vedere le due lettere all'Harvey nelle sue Observationes anatomicae et epistolae medicae ex schedis posthumis selectae et editae a Th. Bartholino, Hafniae 1664, 96-100.

47 Dalla prefazione citata alla nota 44.

48 «neque ego viri consensum obtinere valui, multa cum eo disserens, anno 1638 . quo in aedibus ipsius, humanissime exceptus, per menses quatuor commoratus sum.» Dalla prefazione citata alla nota 44.

49 In data 19 maggio 1636, «cum excellentissimus dominus Paullus Marquard Slegel Hamburgensis, philosophiae et medicinae doctor, inclytae nationis Germanicae artistarum pro tempore bibliothecarius et consiliarius Bohemicus, Romam proficisci constitutum haberet, successores proposuit ...», $399 \mathrm{degli}$ Acta citati nella nota 41.

50 Trascritto a cc. 26-42 del ms. Sloane 2882 del British Museum.

51 Ms. Sloane 2882, c. $26^{\text {r }}$.

52 T. Renda, L'insegnamento delle discipline anatomiche nella «Studiorum Universitas Messanae», Palermo, 1967, 20-23.

${ }^{53}$ Queste considerazioni di P. Castelli meritano di venire esaminate comparativamente a quelle espresse dal Trulli nel brano richiamato dalla nota 108. 
cat, et semper necessario moveatur, si movetur non solo motu trepidationis movebitur, sed fluxionis scilicet de loco in locum: verum quia non datur ultimus terminus ad quem, ubi quiescat; sed motus sit perpetuus, sequitur necessario moveri motu circulari: alias non esset perpetuus. Videtur praeterea dominatio sua cum Harvejo periodorum febrilium causam assignare istam sanguinis circulationem. At, nec equidem medicamenta carpo applicata febrem aliquando finire, nego. Vidi enim saepe, sed in meo Antidotario agens philosophice de Opiatis cum Hippocrate dixi, narcotica febrem finire, eo quod quietem sanguini inducant, et spiritus, qui sunt in motu, quiescere faciant: quasi congelent. Fortasse istum motum spirituum Harvejus circulationem sanguinis vocarit, et de opinione mihi ignota satis. Libentissime quidem ejus opera legerem; sed in has remotas regiones libri novi raro accedunt. Certe Anatome plurimum juvat Medicum, adeo ut Medicum Anatomes ignarum, Medici nomine indignum reor.

Altra testimonianza ci viene da una coppia di lettere scambiate fra lo Schlegel e il Severino. Della missiva dello Schlegel conosco soltanto un frammento $^{54}$, non datato: ed è la formulazione di due problemi chirurgici, in cui sono evidenti i cenni all'ambiente romano. Primo problema è la medicazione rara delle ferite, magnificata anche da taluni chirurghi romani («Ex Chirurgis Romanis aliqui hanc methodum magnifaciunt»). Più indicativa è nel brano relativo al secondo problema (svuotamento dell'essudato pleurico) la seguente affermazione: «Vidi enim hac hyeme Romae ${ }^{55}$ ita saevientem in aliquos hunc morbum ....»

La responsiva del Severino allo Schlegel ${ }^{56}$ è datata «Napoli, 15 maggio». Che fosse indirizzata a Roma ${ }^{57}$, lo possiamo supporre in base al riferimento a due medici romani, e in particolare a Nicola Larche (1602-65) ${ }^{58}$, contenuto nella parte conclusiva; ma ancor più indicativa è la parte iniziale della lettera:

54 Conservato nel ms. Lancis. 70 , c. 55 (= pp. 78-79), dove precede, quindi, immediatamente la lettera indicata nella nota 57.

55 Il corsivo è nostro!

56 Ms. Sloane 2882, cc. 30-31 , donde la lettera fu trascritta da L. Amabile nelle cc. 183-184 del ms. XI. AA. 35 della Biblioteca Nazionale di Napoli.

Tralascio un'altra lettera del Severino allo Schlegel a Jena, datata Napoli, 4 maggio 1639, e conservata nel Ms. Sloane 2882, c. 27; nel ms. Lancis.69, c. 138 ${ }^{\mathbf{r}}(=433)$; e nel ms. Lancis. 71, c. 404 (=1650).

57 Tale indirizzo figura nella copia, parziale e scorretta, di questa lettera conservata nel ms. Lancis. 70 , cc. $56-57^{\mathrm{r}}$ (= pp. 80-82).

${ }^{58}$ Questo chirurgo, francese di nascita, fiorì a Roma, dove acquistò grandissima fama. Lavorò all'Ospedale della Consolazione, dove ebbe come allievo Guglielmo Riva (162777), e all'Ospedale di San Giacomo degli Incurabili. Fu chirurgo pontificio, e il 7 gennaio 1655 imbalsamò la salma di Innocenzo X. Cfr. [G. Marini,] Degli archiatri pontificj, Roma 1784, 1, xlij-xliij; P.SAvio, Ricerche sull'anatomico Guglielmo Riva, Bollettino storico-bibliografico Subalpino 66 (1968) 229-267. 
Duplici me laetitia compleverunt optatissimae literae tuae, et quod restitutum te morbo sentiam gravi, et quod mei memoriam et amorem sincere colas: qua de re plurimum humanitati tuae me debere profiteor. Debeo etiam atque ago gratias immortales et quod coniunctos affatim mihi conserves amicos (inter hos maximus Trollius) ${ }^{59} \ldots$

Un chiarimento prezioso al brano e al nome testè riportati in corsivo, ci viene dalla seguente lettera ${ }^{60}$ (Figura 1$)$ :

Perillustri, et Clarissimo Viro D. Marco Aurelio Severino Domino Suo Colend ${ }^{\mathrm{mo}}$ Ioannes Trullius S.P.D.

Talem me tibi exposuit Marquardi Humanitas, qualem fortasse minime experieris. Vir ille in amicos officiosissimus, de me, quem diligit, testimonium dixit; Tu testem mearum partium admisisti. Non patitur amicitia ut amici Viri condemnem testimonium; immo ut avidis ulnis excipiam beneficium praecipit urbanitas. Utrique debeo ingentes gratias; Tibi vero praesertim, qui praeter admissam de me opinionem honestam, me insuper pretiosissimo munere donasti, atque utinam uti tuas doctissimas locubrationes de recondita abscessuum natura ${ }^{61}$, non legi modo, sed avidissime perlegi, aut potius devoravi, ita explicare coram te possem, quam admirer facilem methodum, nitidum stijlum, ac perspicacem doctrinam, quibus susceptam materiam examinas, explicas, exponis. $\mathrm{O}$ mihi felicem Marquardi amicitiam, quae te mihi conciliavit amicum, ac me fortunatum si tanto viro potero quandoque gratificari. tibi ac tuae virtuti vivam, Severine Doctissime: sed si me amas inter tibi devinctissimorum nomina meum quoque nomen adscribens, mea utere opera ac Vale.

Romae VII Kal. novemb. A.S. mDcxxxvi.

Post has exaratas incidit in febrem Sleghelius noster non sine magno vite discrimine ${ }^{62}$, unde responsum hoc meum tardius ad te perveniet, expectabam nanque donec ille convalesceret, sed cum nimis hoc differatur has ad te mitto atque ipsius quoque nomine salvere iubeo. iterum vale.

Lo Schlegel era presente a Roma almeno dall'autunno 1636, fu colpito in tale stagione da una grave e protratta malattia febbrile, e svernò nella Città Eterna. Se a ciò si aggiunge la sua disponibilità «publicis dissectionibus ${ }^{63}$ » con cui dimostrare la dottrina circolatoria, possiamo proporre in via ipotetica la sua identificabilità col «medico todesco» le cui anatomie sono ricordate con tanto entusiasmo dal Magiotti ${ }^{64}$ nell'aprile del 1637. Questa ipotesi ci apparirà meglio plausibile, quando avremo approfondito la figura del chirurgo Giovanni Trulli (1598-1661), autore della lettera testè riportata.

${ }^{59}$ I corsivi sono nostri!

${ }^{60}$ La lettera, autografa, è conservata nel ms. Lancis. 13, c. $328(=348)$.

${ }^{61}$ Il De recondita abscessuum natura del Severino era apparso a Napoli nel 1632.

62 Il corsivo è nostro!

${ }^{63} \mathrm{Si}$ veda il brano richiamato dalla nota 44 .

${ }^{64} \mathrm{Si}$ veda il brano richiamato dalla nota 26. 
Derflhuthi, et Carittimo Viro . Mares Anclio Sevenino Poño fus Glend?

Joannes Trulling S. P. $\varnothing$.

Talem me tibi exporiet Martuant. Armanitap, qualem

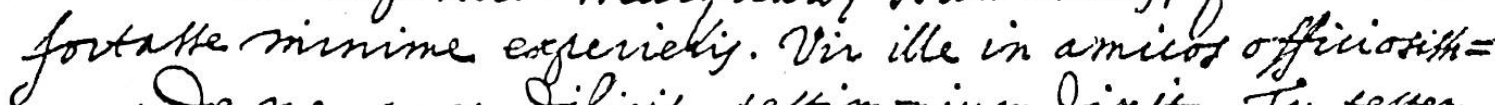
mu, De me, quem Siligit, tettimonium bixit. Fie tertem

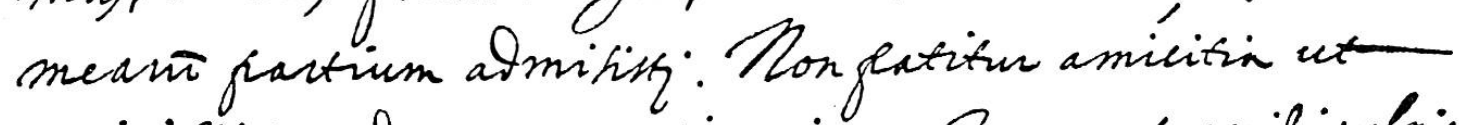
amici vin contemnem tevtinonium. Frmon ux acidijulny S. eacigiam beneficium precifit urbanity. Vtrique bebeo

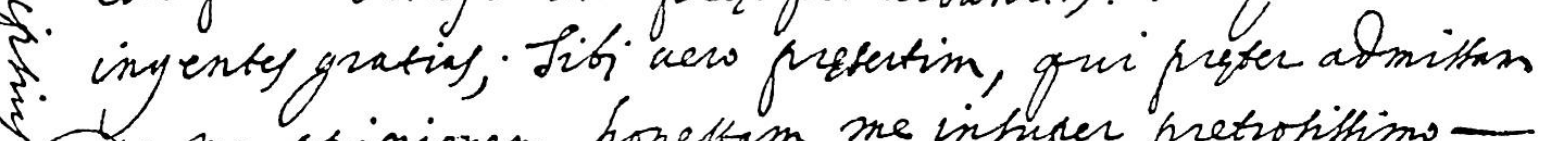
Che me opinionem honetram, me infuger fretrotittino-

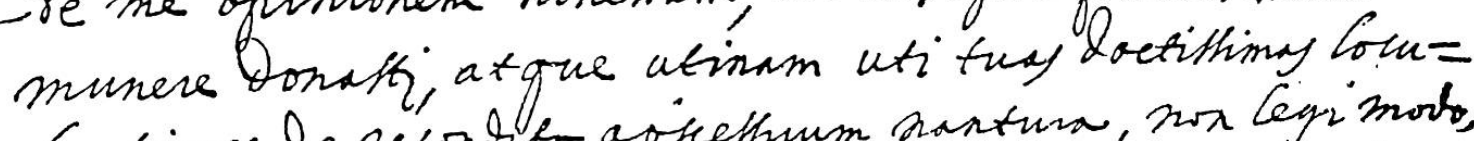

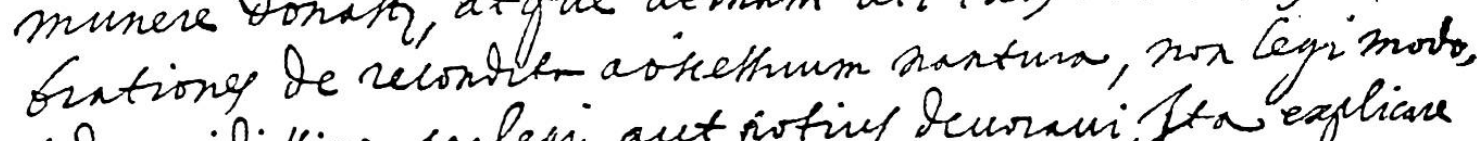
tel avidittime perlegi, aut poting beurani, fer equlicase coum te frottem, quam arminer facilem netholum, nitioñ

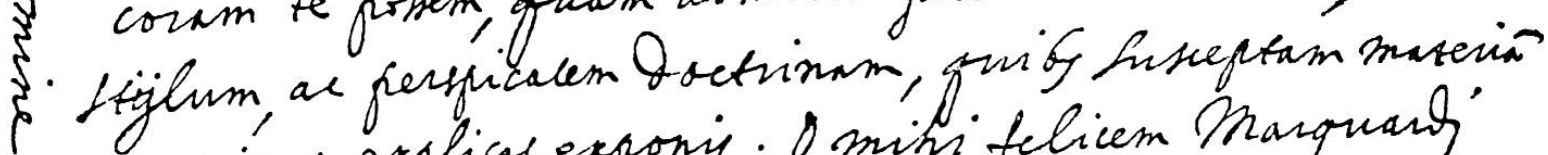

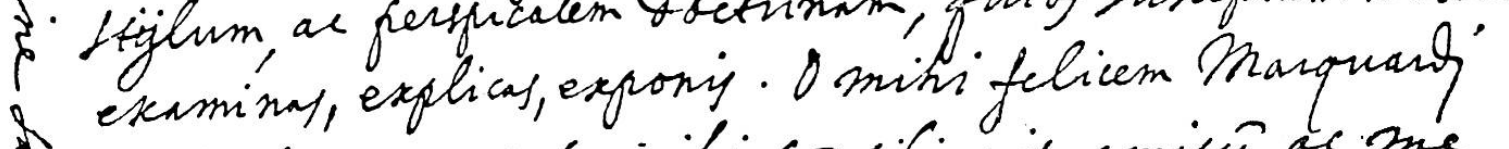
is amicition, que te initi conciliauit amieci, ac mes

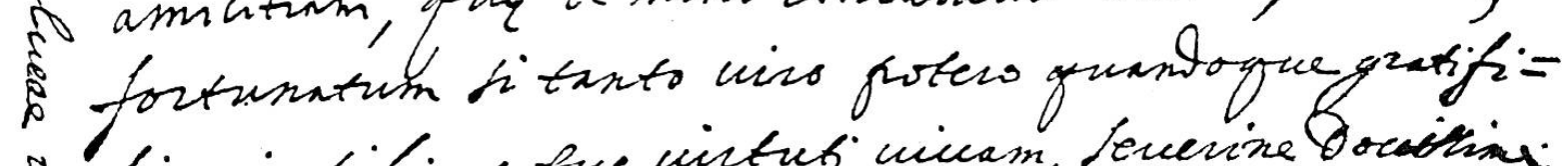

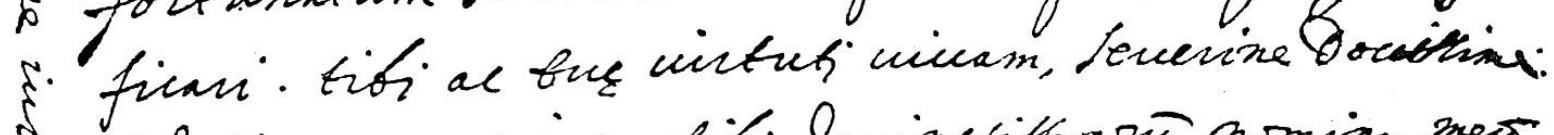

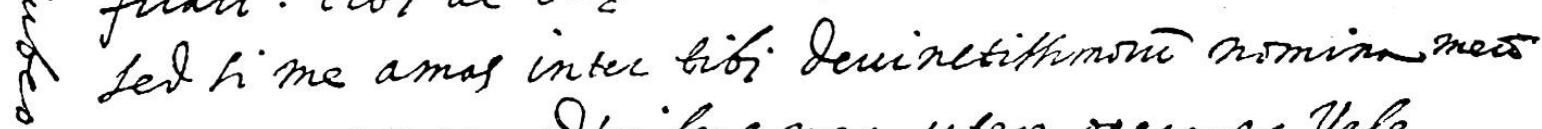
f. quofne nomen attenibens, mea utere operw ac Vale.

हो

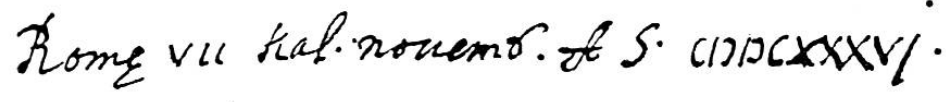

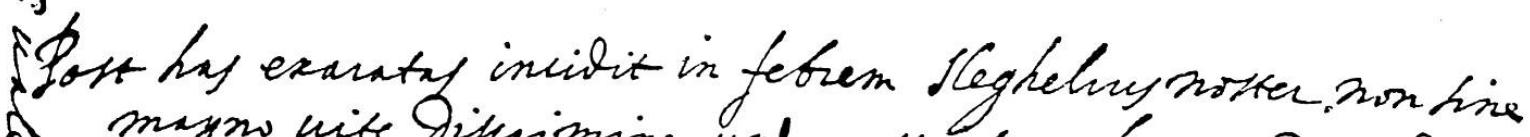

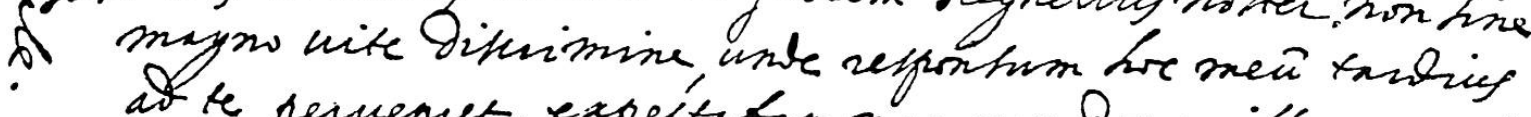

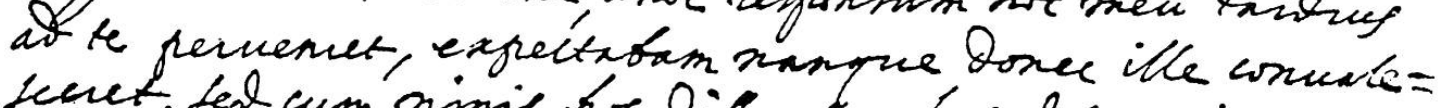

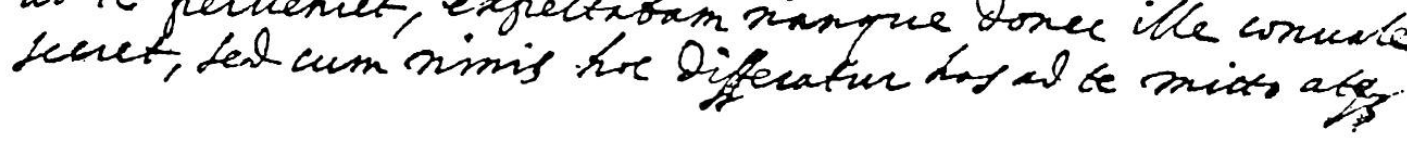

Figura 1. Lettera autografa di Giovanni Trulli a Marco Aurelio Severino, da Roma, 26 ottobre 1636 (ingr. $0,65 \times$ )

Downloaded from Brill.comఠ4/26/2023 ๑4:59:32AM

via free access 
4. G. Trulli, chirurgo «a' servizi del Sr. Card! Barberino». - Sul finire del 1637 il Galilei era divenuto del tutto cieco ${ }^{65}$. Il 23 gennaio 1638, Pier Battista Borghi ${ }^{66}$ gli inviò da Roma un consiglio confacente al suo caso, presentando con queste parole il chirurgo che glielo aveva dettato:

Trovasi in Roma a' servizii del $S$ r. Card! Barberino ${ }^{67}$, con trattenimento non ponto da cortigiano, il Sigr Giovanni Trullio, il quale, dalla sua patria di Veruli passatosene in Francia, ha colà fatto studio particolare nella chirurgia, con tale successo che ha fatto più tosto miracoli che cure in Francia, in Genova et in Roma, et ne fa del continuo. Ha in manco di due anni qui in Roma ${ }^{68}$ cavata la pietra a ventisei huomini, de' quali nessuno è morto e tutti ora godono intiera sanità; il che dico solo a V.S. molto Illre per darle un saggio del valore di questo huomo. Io l'ho conosciuto di là da' monti, e qui in Roma passiamo strettissima familiarità ...

Il Galilei inviò a Roma una relazione, purtroppo non pervenutaci, sul proprio stato oftalmico, in base alla quale il Trulli potè stendere un consulto $^{69}$ in piena regola. Questo consulto, trasmesso dal Borghi all'interessato il 20 febbraio $1638^{70}$, costituisce oggi il più importante documento medico sulla cecità del Galilei ${ }^{71}$. Di qui, l'interesse portato alla figura del Trulli da

65 Si veda il brano richiamato dalla nota 1.

${ }^{66}$ Le opere di G.G. 17, 263.

67 Il corsivo è nostro! Dal $1^{\circ}$ settembre 1636 al 24 maggio 1641 il card. F. Barberini provvide a far pagare semestralmente il canone d'affitto per l'abitazione del Trulli. Cfr. Biblioteca Vaticana, Archivio Barberini, Armadio 42, Mandati di Francesco Barberini 1630-1636, 1637-1641, Numeri 4979 (1 ${ }^{\circ}$ settembre 1636), 5512 (25 agosto 1637), 5597 (4 novembre 1637), 5810 (31 marzo 1638), 6114 (11 novembre 1638), 6299 (2 marzo 1639), 6631 (8 settembre 1639), 6946 (14 marzo 1640), 7433 (2 gennaio 1641), 7691 (24 maggio 1641). Debbo questa notizia - al pari di quelle indicate nelle note $68,72,89,90,94$ e 128 - alle diligenti e sagaci ricerche d'archivio eseguite dalla Signora Jeanne Bignami Odier (Roma), alla quale desidero esprimere la mia profonda e ammirata gratitudine.

${ }^{68}$ Il corsivo è nostro! La casa abitata dal Trulli si trovava in Via de' Cappellari «in regione Arenulae» (Arenella), come risulta da un compromesso passato col fratello Bernardino in data $1^{\circ}$ settembre 1637 (notaio A.C.Domenico Buratto; Archivio di Stato Roma, Archivio dei Notai, vol. 1375, cc. 780 e 815). Il domicilio di Via de' Cappellari è confermato anche successivamente (Archivio del Vicariato, San Lorenzo in Damaso, Stato d'anime). Debbo anche queste notizie alla cortesia della Signora Bignami (cfr. nota 67).

${ }^{69}$ Le opere di G.G.19, 552-554.

70 Le opere di G.G.17, 298.

71 F. Grondona, In tema di eziogenesi della cecità di Galileo, Atti del Simposio «Galileo nella Storia e nella Filosofia della Scienza» (Firenze-Pisa, 14-16 settembre 1964), Firenze 1967, 141-154.

${ }^{72}$ Le opere di G.G.20, 549: "Trullio Giovanni, di Leonardo, nacque in Veroli nel 1598. Soggiornò a lungo in Francia e vi si perfezionò nell'arte chirurgica, acquistando gran- 
Antonio Favaro (1847-1922), al quale dobbiamo un primo profilo biografico $^{72}$.

L'immacolata casistica litotomica «in manco di due anni qui in Roma» va ricollegata al chirografo 3 agosto $1636^{73}$ con cui Urbano VIII assicurava il

dissima riputazione specialmente come operatore di litotrizia. Tornato a Roma, fu chiamato a leggere chirurgia nella Sapienza dal 1637 al 1643, e godè del favore dei pontefici Urbano VIII ed Alessandro VII. Non deve confondersi con l'omonimo suo nipote, che fu pure lettore di anatomia e di chirurgia nella medesima Sapienza. Morì a Roma il 27 dicembre 1661.»

La data di morte è confermata dal ritrovamento dell'atto di morte (Archivio del Vicariato, Liber defunctorum Ecclesiae Sancti Laurentii in Damaso 1644-1672, c. $189^{\mathrm{v}}$ ): «Die 27 hora noctis $2^{\text {a }}$ circa: D. Joannes Trullius fil. $q^{m}$. Leonardi Verulan. ann. 62 doctor celebris ac chirurgus maximorum Pontificum Urbani VIII et Alexandri VII in domo propria in via vulgo a Cappellari in Communione Sanctae Matris Ecclesiae Deo animam reddidit, Sacramentis omnibus ecclesiasticis rite praemunitus ultimae Poenitentiae a P.Hieronymo Barnabeo die $25 \mathrm{~S}^{\text {mo }}{ }^{\circ}$ Viatico refectus die 26 a R.D. Jo. Vicar. et ab eodem dicta die $26 \mathrm{~S}$. Olei unctione roboratus, cuius corpus, post solemne funus in hac ecclesia peractum, de sero ad ecclesiam S. Mariae Angelorum ex testamenti sui dispositione sepeliendum fuit delatum.»

All'Archivio di Stato Roma (Notaio Capitolino Nicolaus Rignanus, ufficio 6 [28]) è conservato il testamento olografo del Trulli in data 24 settembre 1652 (cc. 325-326 e 339 ), unitamente ai codicilli, pure olografi, in data 24 settembre 1654 (c. 327 ), con la dichiarazione testimoniale (c. $\left.338^{\mathrm{v}}\right)$ : «Ill. et ex ${ }^{\text {us }}$ D. Joannes Trullius fil.q. D. Leonardi Verulanus et Civis Romanus artium medicinae Doctor ...». Il testamento è preceduto (c. 324) dal verbale della «Aperitio Testamenti et Codicillorum ... Die 27. Decembris 1662. a. Nativitate». Privo di discendenti diretti, il Trulli menziona, nel testamento e nei codicilli, i fratelli e i loro discendenti. Ricordo in particolare il fratello Stefano (chirurgo all'Ospedale di Santo Spirito, come attesta H.v.Möinichen a p. 243 dell'opera citata alla nota 97) e Giovanni, figlio di Stefano (che è probabilmente «l'omonimo suo nipote, che fu pure lettore di anatomia e di chirurgia nella medesima Sapienza » ricordato dal Favaro; cfr., più avanti, la nota 84 ).

Anche i documenti segnalati nella presente nota sono stati cortesemente ritrovati dalla Signora Bignami (cfr. nota 67).

${ }^{73}$ La seguente trascrizione parziale è stata da noi condotta sul documento conservato all'Archivio Storico Capitolino (Credenzone VI, t. 52, c. 31). Per il testo integrale si veda F.M. Renazzi, Storia dell'Università degli Studj di Roma detta comunemente la Sapienza, Roma 1805, 3, 244 (e 96). Nella stessa opera, a p. 252, il Renazzi riporta un altro chirografo, particamente identico nella parte per noi sostanziale, datandolo, però, 3 luglio 1633. All'Archivio Storico Capitolino (Credenzone VI, t. 52, c. 161 ${ }^{\mathrm{v}}$ ) è conservato altro chirografo del 15 maggio 1655 con cui Alessandro VII - facendo esplicito riferimento al chirografo di Urbano VIII in data 3 agosto 1636 - ribadisce che "per la detta somma di scudi duecento non si possino impiegare in altro, ma restino, et debbiano restare sempre fermi per le provisioni et augumenti delli Lettori ...». 
reperimento della parte principale dei fondi perchè il Trulli potesse impartire, vita natural durante, l'insegnamento della litotomia e dei grandi interventi della chirurgia dell'epoca ${ }^{74}$ :

... detti scudi 200 durante la vita di Giovanni Trulli nostro suddito da Veroli educato in Francia se gli assegnino in parte della provisione da Noi destinatagli, acciò resti in Roma a benefitio et utile publico con obligo di operare con la sua professione, et impiegarsi gratis per li Poveri ogni volta che da quelli ne sarà ricercato, e d'instruire nel luogo che li sarà assegnato dal Rettore dello Studio chiunque verrà a fare l'operationi più difficili della Chirurgia, e specialmente a levar la Pietra della Vessica, acciò con progresso di tempo per suo mezzo si stabilischi in Roma detta peritia.

Negli elenchi dei lettori dell'Archiginnasio Romano ${ }^{75}$ il nome del Trulli compare alla fine del rotulo per il 1636: «M. Io.Trullus Verulanus in Chirurgia extra ordinem in diebus vacantibus » con la retribuzione di 300 scudi $^{76}$. La voce è ripetuta, con identica retribuzione, nei successivi rotuli per gli anni $1637^{77}, 1639^{78}$ e $1640^{79}$ : e la stessa lettura ci è confermata da Thomas Bartholin (1616-80 $)^{80}$ in data 4 dicembre 1643. Nel successivo rotulo per il 1645 l'insegnamento del Trulli è scomparso. Una lettura «in Chirurgia et Anatomia, hora vespertina » assegnata a un Giovanni Trulli con la retribuzione di 80 scudi compare nel rotulo del $1658^{81}$ e si ripete nei rotuli per il $1661^{82}$ e per il $1664^{83}$, continuando, quindi, oltre la morte del Giovanni Trulli che a noi interessa ${ }^{84}$.

${ }^{74}$ Fra i quali rientrava - e lo ricordo a proposito del consulto del Trulli a favore di Galileo anche l'operazione della cataratta.

75 Arch. di Stato Roma, Arch. dell'Università di Roma, Parte II, Collegio medico, Busta 94.

76 c. $115^{\mathrm{r}}$.

77 «In Chirurgia extr. Ord. in diebus vacant. M.Ioannes Trullus Verulanus per annum prof. est », c. 124.

78 «In Chirurgia extra ord., hora matutina M. Ioannes Trullus Verulanus prof. per an. 3.», c. $137^{\mathbf{v}}$.

79 «In Chirurgia extra ordin., hora matutina M. Ioannes Trullus Verulanus profes. est per an. $4 »$, c. $146^{\mathrm{r}}$.

80 Epistolarum medicinalium a Doctis vel ad Doctos scriptarum Centuria I. et II., Hafniae, 1663, p. 191 (Cent. I, epist. XLIII «De Medicis Romanis »): «Diebus vacantibus docent... D. Jo. Trullus Verulanus in Chirurg. extr. de Lapide Vesicae h. 16.».

81 «In Chirurgia et Anatomia, hora vespertina: M. Ioannes Trullus Verulanus prof. per annum », c. $230^{\mathrm{v}}$.

82 «In Chirurgia et Anatomia, hora vespertina: M. Ioannes Trullus Romanus prof. per an. $4 . »$, c. $252^{\mathrm{v}}$.

83 «In Chirurgia et Anatomia, hora vespertina: M. Ioannes Trullus Romanus prof. per an. 7.», c. $270^{\mathrm{v}}$.

${ }^{84}$ Si tratta evidentemente dell'omonimo nipote, che, come ho indicato nella nota 72 , era stato individuato dal Favaro. Giovanni Trulli jr. «insegnò poi la Medicina teorica ... e finalmente la Medicina pratica sin'all'anno 1700 » (RenazzI, Storia dell'Università di Roma 3, 189). 
Quanto oculatamente Urbano VIII riponesse la sua fiducia conferendo al Trulli l'insegnamento della chirurgia operatoria, risulta dalla testimonianza diretta di un allievo: l'aretino Anton Filippo Ciucci ${ }^{85}$, fierissimo della propria squisitezza di litotomo "post diuturnam decem annorum praxim Romae apud Excellentiss. D. Ioannem Trullium seniorem, cuius vices immerito sustinui in Xenodochio Apostol.S. Spiritus in Saxia per annos quinque ${ }^{86}$.

Nel «Catalogo ovvero serie cronologica de' medici pontificj» ${ }^{87}$ Giovanni Trulli compare nel 1642 quale chirurgo dell'infermeria di palazzo; scompare nel conclave del 1644 e durante il pontificato di Innocenzo $\mathrm{X}^{88}$; e ricompare nel conclave del $1655^{89}$ e sotto il pontificato di Alessandro VII.

Il 29 luglio 1644 il Trulli imbalsamò la salma di Urbano VIII ${ }^{90}$. Nel corso della eviscerazione preliminare furono osservati «in sinistro cordis ventriculo os triangulare, literam $\mathrm{T}$ referens, et quinque calculi in vesica fellea, singuli magnitudinis nucis avellanae, et in renibus multi lapilli exigui». Questa descrizione ${ }^{91}$ ci è stata lasciata da Thomas Bartholin $(1616-80)^{92}$, di cui sono noti i rapporti diretti col Trulli ${ }^{93}$. Quindici anni più tardi, Henrik

${ }^{85}$ Per notizie su questo chirurgo è da vedere l'introduzione a A.F. Crucci, L'Ospidale di Parnaso a cura di Bruno Zanobio, Milano 1962.

86 A. Ph. Crucci, Promptuarium chirurgicum, Macerata 1679, 141. Il Ciucci riporta inoltre la ricetta dell'unguento impiegato dal Trulli nella cura delle scottature (p. 136) e la guarigione, da lui osservata attorno al 1651, di una ferita tracheale «in quadam Meretrice Brigida dicta», curata «ab Ex. D. Ioanne Trullio cum sutura Aureo filo peracta» (pp. 137-138).

87 [MARINI], Degli archiatri pontificj 1, xlij-xliij.

${ }^{88}$ Dopo la morte di Urbano VIII, i protetti dei Barberini caddero in disgrazia.

89 Già in periodo di sede vacante gli fu attribuito un assegno mensile di 100 scudi (Archivio di Stato, Camerale I, no 954, c. $137^{\mathrm{r}}$ ). Per cortese comunicazione della Signora Bignami (cfr. nota 67).

90 Per questo lavoro gli furono versati 25 scudi in data 3 agosto 1644 (Archivio di Stato, Camerale I, no 954, c. $62^{\mathrm{v}}$ ). Per cortese comunicazione della Signora Bignami (cfr. nota 67).

91 Sostanzialmente concordante con quella contenuta nel Memoriale di Cassiano dal Pozzo e riportata a pp. 185-186 del lavoro citato alla nota 8.

92 Th. Bartholin, Historiarum anatomicarum rariorum centuria I. et II., Hafniae 1654, 243-244 (Centuria II., Historia XLV.: «Os in Corde Urbani VIII.»).

Il Bartholin ricordò brevemente il reperto anche in Epistolarum medicinalium a Doctis vel ad Doctos scriptarum Centuria I. et II., Hafniae 1663, 273 (Centuria I., Epistola LXVI: «Dubia de Circulatione Licetiana») e nella Anatome ... quartum renovata, Leida 1673, 394 (lib. II, cap. VII «de cordis partibus in specie»).

Il racconto del Bartholin circa l'ossificazione nel cuore di Urbano VIII è anche ricordato da G. B. Morgagni, De sedibus et causis morborum per anatomen indagatis, Venezia 1761, 1, 292 (= Epist. 27, Art. 19).

93 Si veda, in proposito, la lettera del Trulli al Severino datata 29 gennaio 1644 e richiamata dalla nota 113. 
van Möinichen (1631-1709) ${ }^{94}$, egli pure danese, comunicherà a Th. Bartholin il reperto interessante la storia dell'arteriosclerosi ${ }^{95}$ - osservato nella salma del cardinale Cornelio Melzi, alla cui dissezione era stato chiamato, il 21 gennaio 1659, «cum Cl. Viro Tuique studiosissimo D. Johanne Trullio Medico-Chirurgo Pontificio». Racconta poi Giovanni Maria Lancisi (1654-1720) ${ }^{96}$ che sotto il pontificato di Alessandro VII (1655-67) «cadavera quamplurima repente mortuorum adaperta fuerunt a Joh. Trullio Chirurgo Pontificio, qui, fere in omnibus, vasorum vel intra pectus, vel intra cranium rupturas comperit».

Interessanti notizie sull'attività clinico-chirurgica e medico-legale del Trulli ci giugnono ancora dal Möinichen, nelle lettere a Th. Bartholin ${ }^{97}$, nonché da tre colleghi del Trulli all'Università di Roma. Uno di essi è Domenico Panaroli (m. 1657 $)^{98}$. Il secondo è Benedetto Castelli, che già conosciamo quale professore di matematica. In una lettera del 20 settembre $1638^{99}$, che costituisce un importante documento per la storia del termometro Galileiano

94 Th. Bartholin, Epistolarum medicinalium a Doctis vel ad Doctos scriptarum Centuria I. et II., Hafniae 1663, 720-726 (Centuria II., Epistola X CVII. "Observationes Anatomicae in cadavere Cardinalis Meltij, et Nattae Sectio »: la lettera è datata Roma 12 novembre 1659). - Al Trullio dobbiamo, inoltre, la storia anatomico-medica del cardinale Orazio Giustiniani (1580-1649), che fu anche prefetto della Biblioteca Vaticana. La sezione, praticata il 25 luglio 1649 «sì per preparare il cadavere alla conservatione, come si suole nelli grandi, come anco mettere apertamente in chiaro le cause dell'improvvisa rovina", dimostrò infatti un "tumore nel collo della vessica» con vescica a colonne e infezione ascendente delle vie urinarie. Questa storia, essa pure gentilmente comunicatami dalla Signora Bignami (cfr. nota 67), trovasi all'Archivio di Stato Roma, Fondo Giustiniani, Busta 24, mazzo $\mathrm{P}, \mathrm{n}^{\circ} 8$.

95 L. Belloni, Introduzione storica alla patologia dell'aterosclerosi, in [C. CAvallero edit.] Aterosclerosi umana e sperimentale, Milano 1956, 25-66.

96 G.M.Lancisi, De subitaneis mortibus, Roma 1707, lib. 2, cap. 3, art. 7.

${ }^{97}$ Henrici à Möinichen D. Medici Regii Hafn. Observationes Medico-Chirurgicae, Missae ad Thomam Bartholinum. Stanno in: M. Lyser, Culter Anatomicus ... Secunda hac editione Observationibus nonnullis Variorum aucta cum praefatione Th.Bartholini, Hafniae 1665, 241-256. Si vedano le osservazioni: III. «Abscessus cruris per urinam expurgatus» (242243); XIII. "Vulvae clausae apertio» (247-248); XIV. "Aneurisma sponte ruptum» (248-249); XXIV. «Nova Herniae curatio» (256). L'osservazione III fu compiuta nell'Ospedale di Santo Spirito: e il Möinichen ci dà notizia di uno Stefano Trulli, fratello di Giovanni, e chirurgo ordinario dello stesso ospedale (sul quale si veda la nostra nota 72). Apprendiamo inoltre che G. Trulli si proponeva di pubblicare una raccolta di osservazioni e un trattato sull'aneurisma. Altra osservazione interessante è la «Nattae Sectio» descritta dal Möinichen nella lettera citata alla nota 94 .

98 Il quale descrive una perizia di venereologia forense compiuta in associazione col Trulli; cfr. D. Panaroli, Iatrologismorum seu medicinalium observationum pentecostae quinque, Roma 1652, 355 (Pentec. V, observ. L «de hymene»). Nell'«Arcanorum faciculus secundus » portato in appendice a questo volume (pp. 436-437 e 442) il Panaroli riconosce di non essersi ancora fatta un'opinione personale sulla circolazione del sangue e di attendere, a questo fine, alcuni opuscoli promessigli da Cassiano dal Pozzo, da noi ricordato nel brano richiamato dalla nota 8 .

${ }^{99}$ Le opere di G.G.17, 377-380. 
(termoscopio), il Castelli discute il comportamento, fisicamente paradosso, dell'intestino prolassato, il cui volume aumenta ${ }^{100}$, mentre dovrebbe diminuire per effetto del raffreddamento. Lo spunto alla discussione era venuto al Castelli dalla « cura, veramente maravigliosa, fatta dall'Eccl ${ }^{\mathrm{mo}}$ Sig. Giovanni Trullo di quel povero che a' mesi passati, essendo stato ferito nel ventre, gli erano per la ferita uscite gran parte delle budella, e perciò era da acerbissimi dolori tormentato; nè si trovando modo da' medici nè da' cerusici di rimetter al suo luogo quegli intestini, stante che dopo essere uscite dal lor luogo del ventre si erano stranamente rigonfiate, fu chiamato il sudetto Sig. Trullo, il quale, veduto che ebbe il paziente, con gran franchezza e risoluzione prese un ago, e pungendo in diverse parti quell'intestina, scappando via quel flato rinchiuso, subito sgonfiarono, e facilissimamente furono nel ventre del ferito rimesse; e chiusa la piaga e dopo, conforme all'arte curata, l'infermo si ridusse nella pristina sanità».

Molto importante, infine, è la testimonianza di Giovanni Benedetto Sinibaldi (1594-1658), lettore «in simplicibus medicamentis necnon in medicina practica extra ordinem », e non tanto perchè ci dà un'ulteriore conferma ${ }^{101}$ dell'abilità litotomica del Trulli, quanto perchè ce lo presenta in veste di paladino della dottrina circolatoria:

Quod si vera esset Hervaei sententia illa de sanguinis circulatione, quam inter alios Romae probat, strenueque defendit egregius medicus chirurgus Joannes Trullus in Romana Academia lithotomiae magister, divina providentia Italiae, et Urbi ex Gallia redditus, ut huic saeculo summe lapidoso consuleretur...

Questo brano - contenuto in un'opera del Sinibaldi apparsa nel 1642 102 figura, nell'elenco cronologico del Weil ${ }^{103}$, quale «first acknowledgment in

100 Ammaestrativo, in proposito, è il racconto di Ambroise Paré (1510-90) in Euvres complètes par J.-F. Malgaigne, Paris 1840, 2, 107.

101 Laddove auspica che venga messa a punto sull'animale da esperimento la litotomia renale, onde poterla poi praticare nell'uomo: «Vos itaque Galli eximij in Arte Viri, Vos Larghe, ac Trulle Urbis Coryphaei contendite, brutorum periculo sectionem experimini, tanto beneficio humanum genus ne defraudetis ...», Hippocratis Coi Medicorum Principis $A N T I P H \Omega N \Omega N$ libri quinque ... auctore Io. Benedicto Sinibaldo, Roma, 1650, 318 (Lib. V, ANTIPHSNIA IV.: "An ad calculum educendum liceat Medico sectionem moliri»). L'invocazione del Sinibaldi è riportata, e ulteriormente caldeggiata, dal Panaroli a p. 345 della sua opera citata alla nota 98.

102 Geneanthropeiae, sive de hominis generatione decateuchon, Roma, 1642, col. 637 (Lib. V., Tract. I., Cap. XIV: «An seminis materia sit cambium, an vero sanguis ille secundarius, qui dicitur anonymus»).

Un accenno del Sinibaldi alla dottrina della circolazione trovasi anche a p. 29 della sua opera qui citata alla nota 101: «Denique in eum finem ex Hervaei sententia sanguinis circulatio instituta a natura fuit, ut nimirum calorem, et spiritus, quos per corpus excurrens amittit, in corde, cum remeat, repararet» (Lib. I, ANTIPHSNIA V.: «Sitne sanguis natura calidus, necne»).

103 E. WeIL, The Echo of Harvey's De Motu Cordis (1628) 1628 to 1657, J. Hist. Med. 12 (1957) 167-174. (cfr. 170); KEYNEs, The Life of W.H., 451. 
Italy » della dottrina di Harvey, e costituisce anche per altri, e importanti, autori ${ }^{104}$ uno dei punti fissi nella storia della dottrina della circolazione del sangue in Italia.

5. G. Trulli e la dottrina della circolazione: testimonianze nel suo epistolario col Severino $e$ in opere del Severino. - L'attivo interesse del chirurgo «a' servizi del S. Card! Barberino» per la dottrina circolatoria non ci meraviglia affatto al lume delle notizie ecologiche che siamo andati raccogliendo su di lui, a partire dalla sua stessa amicizia con lo Schlegel attestataci dal suo epistolario col Severino. Questo stesso epistolario ci consentirà ora di ribadire il suo interesse per la dottrina circolatoria e di risalire a qualche suo breve scritto sull'argomento.

I rapporti Trulli-Severino, che erano iniziati, pronubo lo Schlegel, nel tardo 1636, subirono una riaccensione nel gennaio 1641, quando un comune amico, che aveva visitato a Napoli il Severino, rientrò a Roma, latore di una lettera ${ }^{105}$ al Trulli con l'invito «si quid rariorum aut novarum observationum habes, has amico communica».

Il Trulli rispose il 9 febbraio $1641^{106}$ dicendosi disposto a corrispondere all'invito, e lasciandoci, al tempo stesso, un'importante conferma della sua attività di paladino della dottrina circolatoria:

... de raris autem meis observationibus quasdam ex Harvei nostri mente de cordis motu, viri equidem viventium peritissimi Anatomici habeo. cuius cum doctrinam in urbe palam fecissem, de sanguinis circuitu ${ }^{107}$, aliis addiscendi aviditatem aliis antiquae nec non vulgari medicinae nimis adhaerentibus pertinaciam attuli. quasi vero re in medica potius aliorum authoritati quam caeterorum rationi praestanda esset fides, quam non secus ac rem novam respuerunt. In eandem mihi videtur Hippocrates ivisse sententiam ubi ait: «Venae per corpus diffusae spiritum, fluxum, et motum exhibent ex una multae germinantes atque

104 W.PAgel e F.N.L. Poynter, Harvey's Doctrine in Italy: Argoli (1644) and Bonaccorsi (1647) on the Circulation of the Blood, Bull. Hist. Med. 34 (1960) 419-429 (cfr. 420); W.PAgeL, William Harvey's Biological Ideas, Basel/New York 1967, 60 e 350.

105 Ms. Lancis. 71, c. 345; e ms. Lancis. 74, c. 268 (= 583). In entrambi gli esemplari, la lettera, pur portando due volte nel testo il vocativo «Trulli», è indirizzata «Joanni Trulliero Romam», forse per una confusione con quel «Giuseppe Trullier Med. del SS. Sac. » citato dal Marini in Degli archiatri pontificj, 1, xlij, e ricordato anche dal Severino in De recondita abscessuum natura, Napoli 1632, 141 del IV libro ( De novissime observatis abscessibus», e precisamente nella storia anatomico-medica di Marcello Sacchetti, aggiunta alla fine di tale libro).

106 Ms. Lancis. 13, c. 329 (= pp. 349-350).

107 Il corsivo è nostro! 
haec una unde oriatur ac ubi desinat non scio: circulo enim facto, principium non invenitur $\gg .{ }^{108}$ cuius mentem ab Hyppocrate non dissidere infero. nec proinde reiciendam exqua et multas expertus et insolentes observationes, ubi per tempus licuerit communicabo tibi.

Il $1^{0}$ aprile $1642^{109}$, a più di un anno di distanza, il Severino ricorda la promessa, e facendo pressante riferimento all'Harvey, richiede le osservazioni del Trulli sulla circolazione del sangue:

Annus est iam, a quo tempore (si recte memoria tenes, mea me utique non fallit) promiseras missurum te novas tuas observationes de cordis sese moventis germanis caussis, deque sanguinis ab habitu corporis ad intima recursu: quam rem acutissimi Vir ingenij Gulielmus Harveus ${ }^{110}$ hac aetate nostra subtilissimis ostensionibus explicatam publicavit; rectissime tu quidem, et sagaciter Trulli primum ab Hippocrate adversam indicasti postremis tuis ad me literis. Tempus quidem abunde fuit, ut meditata consummaveris, maturaverisque. Ut pollicita iam praestes, impense quaeso te rogoque: praesertim quando certiorem facturus sum inclitum Virum mei amantissimum de facie notum Amicum, qui meum hac de re iudicium litteris, et libro missis anxie petit: placitum hoc suum licet Gallis et Cisalpinis plerisque invisum, Romanis tamen Doctoribus, e quibus unus Trullius meus est, itemque Severino, si parva licet componere magnis, Jano Capucio ${ }^{111}$, quem Virum clara fama manet, atque alijs plerisque summe probatum. Age igitur, hunc tibi Auctorem, quem ita colere profiteris, vehementer es devincturus, si quidem in eo te problemate non vulgariter exercitum demonstraris.

Lo scambio epistolare si infittisce nel 1644 - ben 9 lettere! - dopo un viaggio a Napoli compiuto dal Trulli e attestato da una sua lettera in data 29 gennaio $1644^{112}$. Tosto rientrato a Roma - scrive il Trulli al Severino «absque mora doctissimum Bartholinum ${ }^{113}$ quaesivi, et ipse apud me bis fuit ... neuter tamen alterum invenit »: e infatti Th. Bartholin partì alla volta di Napoli senza che il loro incontro fosse possibile. La lettera prosegue con un cenno a Cassiano dal Pozzo ${ }^{114}$ (che, pronubo il Trulli, strinse allora

108 De natura ossium, 11 (LitTré, 9, 182-183). Queste considerazioni del Trulli meritano di venir esaminate comparativamente a quelle espresse da Pietro Castelli nel brano richiamato dalla nota 53.

109 Ms. Lancis. 71 , c. 349 ; e ms. Lancis. 74 , c. $268^{\mathrm{v}}$ (= c. $\left.583^{\mathrm{v}}\right)$.

110 Si veda la nota 16 .

111 Su Giovanni Battista Capucci, membro dell'Accademia degli Investiganti di Napoli, è da vedere M.H.FISCH, The Academy of the Investigators, in E.A. UNDERwood edit., Science, Medicine and History. Essays on the evolution of scientific thought and medical practice written in honour of Charles Singer, Oxford University Press, 1953, 1, 521-563.

112 Ms. Lancis. 13, cc. $330^{\mathrm{r}}-331^{\mathbf{r}}$ (= pp. 351-353).

113 Si vedano i brani richiamati dalle note 80 e 94 .

114 Si vedano le note 8 e 98. 
amicizia col Severino); con le osservazioni del Trulli sull'apparato circolatorio dei serpenti (che verranno letteralmente e integralmente inserite dal Severino nella Vipera Pythia, 1651) ${ }^{115}$; con l'annuncio di un supplemento di osservazioni sullo stesso argomento («Pergam dissecare huiusmodi serpentes, et viperas etiam quando haberi poterunt, ut veritatem opinionis Harvaei demonstrem ex meis observationibus, omniaque tecum communicabo »); e con alcune considerazioni su Ippocrate e la circolazione analoghe a quelle che già conosciamo ${ }^{116}$.

Il Severino ringrazia in data 22 febbraio $1644^{117}$ e sprona il Trulli con l'elogio:

Iamdudum comperta mihi est ingenij tui perspicacitas, iudicijque maturitas in re non solum anatomica, sed et in universa re Medica, qua ingenij solertia et acutissimi Anatomici Gulielmi Harvaei de refluo sanguinis cursu et firmare et locupletare abunde potes.

L'elogio è seguito dalla richiesta di una dissertazione promessa dal Trulli e che il Severino intende inserire in una propria opera: «... anatomicae de Vena salvatella disquisitionis oblitus tam brevi fuisti? ... de qua re et serio te rogaveram Neapoli, et ipse non ambigue promiseras ...»

I solleciti si alternano ${ }^{118}$ alle scusanti, non escluso un disguido postale. Nel settembre 1644 il Severino accusa finalmente al Trulli ricevuta della dissertazione, come apprendiamo dalla sua lettera del 6 dicembre $1644^{119}$ :

Per una mia lettera all'ordinario corriero nel mese di settembre data, rendei a VS. le dovute, $o$ più tosto da me render potute, gratie, sì per l'Anatomica figura, sì per la spositione delle vene del corpo humano, l'una et l'altra dalla ugual sua diligenza et scienza ordinate. Dottrina certo per lo nostro proposto sì ben acconcia et agiata, che non si può più desiderare, di modo che soddisfatto a pieno io ne sono rimaso.

E la lettera De serie venarum, che verrà inserita dal Severino nella Seilophlebotome castigata, sive de Venae salvatellae usu et abusu (Hanau, 1654) ${ }^{120}$ e che così comincia :

115 Vipera Pythia, idest De Viperae Natura, Veneno, Medicina Demonstrationes et Experimenta nova, Padova 1651, 289.

116 Si veda la nota 108.

117 Ms. Lancis. 74, cc. $277^{\mathrm{r}}-278^{\mathrm{r}}\left(=592^{\mathrm{r}}-593^{\mathrm{r}}\right)$.

118 Severino al Trulli, 31 marzo 1644, ms. Lancis. 74, c. 269 (=584).

Trulli al Severino, 10 aprile 1644, ms. Lancis. 13, c. $334^{\mathrm{r}}\left(=356^{\mathrm{r}}\right)$.

Trulli al Severino, 30 aprile 1644, ms. Lancis. 13 , c. $335^{\mathrm{r}}\left(=357^{\mathrm{r}}\right)$.

Severino al Trulli, 14 maggio 1644 , ms. Lancis. 74 , cc. $270^{\mathrm{r}}-271^{\mathrm{r}}\left(=585^{\mathrm{r}}-586^{\mathrm{r}}\right)$.

Trulli al Severino, 8 giugno 1644, ms. Lancis. 13, c. $336^{\mathbf{r}}\left(=358^{\mathrm{r}}\right)$.

119 Ms. Lancis. 10, c. 234; ms. Lancis. 71, c. 217.

120 Pp. 150-153. 
Venarum Anatomicam ostensionem, ex quibus salvatella gignitur, ut petis, mi Marce, ad te mitto. Haec unde ortum ducat, ex quibus ramis, ijque tandem a quo trunco propagentur, ex acclusa pagina patet. Talem autem delineationem hanc meam reperies, qualem ego longissima diligentissimaque inspectione dissectis cadaveribus iuxta naturae ordinem truncorum dispositionem observavi.

La successiva descrizione dell'apparato venoso concorda con la tavola panoramica dello stesso apparato inserita a p. 7, che è forse identificabile con «l'Anatomica figura» inviata dal Trulli al Severino.

«Salvatella ${ }^{121}$ è termine che si vuol derivato dall'arabo «alaseilem» attraverso una sapiente corruzione per cui ne sortì un equivalente di «vena salutis». Il salasso praticato dalla salvatella sinistra trovava, infatti, tipica indicazione nelle malattie da melancolia, in quanto l'evacuazione così ottenuta incideva a livello della milza, centro di tale umore, mentre il salasso praticato alla salvatella destra evacuava dal fegato. Al pari di tutto ciò che si riferiva a un intervento di così fondamentale importanza come il salasso, anche il problema della elezione della salvatella era oggetto di vivaci dibattiti. Per spiegare l'evacuazione di melancolia dalla salvatella, Adriaan van der Spieghel (Spigelius, 1578-1625) ${ }^{122}$ aveva invocato l'esistenza di generose anastomosi fra questa vena e le arterie, anastomosi, a suo avviso, necessarie per soddisfare l'ingente richiesta di sangue più caldo e spiritoso da parte di una estremità, come la mano, che è lontana dal cuore, fonte del calore vitale. L'evacuazione di sangue arterioso, impossibile col salasso dalla piega del braccio, si ottiene invece col salasso dalla salvatella: e l'evacuazione di sangue arterioso è in tanto benefica ai melancolici, in quanto il corredo della milza in arterie è sei volte superiore al corredo venoso. Si tenga presente che la dottrina Galenica offriva allo Spieghel un percorso relativamente breve dalla milza alle anastomosi da lui descritte: arteria splenica, a. celiaca, a. aorta, a. succlavia-ascellare-omerale, diramazioni di quest'ultima fino alle anastomosi con la v. salvatella sin.

Il Trulli ritiene che le anastomosi artero-venose ammesse dallo Spieghel a livello della salvatella, siano troppo fini per essere visibili ${ }^{123}$ :

Atque aliquando disputans hisce de rebus, opificij humani peritissimus Ioannes Trullius Medicus Romanus enunciavit, inquirendarum quidem facile, verum reperiendarum anastomoseon extremarum, cassum futurum omnem laborem, quando capilliformium utrorumque vasorum respondentes coitus et oscula pervidere discernereque lyncei vix oculi queant.

Riferisce il Trulli, nella lettera De serie venarum, che dei due rami cefalico e basilico - in cui la vena ascellare si biforca, «ille caput, hic lienem,

121 G. HyrtL, Onomatologia anatomica, Roma 1884, 323.

122 De humani corporis fabrica, Venezia 1627, 171 (lib. V, cap. VII «Axillaris Venae per brachium distributae seriem proponit»).

123 Seilo-phlebotome castigata, 107-108. Il Trulli è inoltre citato a pp. 12 e 61. 
scilicet sinistri lateris, evacuare dicitur; dextri vero hepar » ${ }^{124}$; ma ignora come si possa spiegare questa diversità di comportamento, data la comune origine di quei rami, che, in ultima analisi, si rifanno al tronco venoso della cava. Quanto alla problematica evacuazione di sangue melancolico dalla salvatella, il Trulli fa presente la dipendenza di questa vena dal tronco della cava, il quale

... sanguinem puriorem tenuioremque atque sinceriorem pro nutritione partium sibi vendicat; porta vero sanguinem crassiorem, ac faeculentiorem, tum ab excrementis cum repurgandum, tum ad viscera alendum continet. Hic igitur sanguis, qui tanquam fex in vino, aut amurca in oleo in propaginibus hujusmodi vasorum stabulatur, nescio qua via per salvatellae sectionem possit educi, ac evacuari. Si id fieri contingeret, oportet sanguinem ex vasis portae remeare in hepar, ex eo per cavam ad cor, deinde ad claves, tandem ad brachium et manum deferri ${ }^{125}$.

In complesso, il Trulli non emerge dalla lettera De serie venarum come un paladino particolarmente agguerrito della dottrina circolatoria. A questa sembra invece far miglior riferimento il Severino, mentre, nella lettera testè citata $^{126}$, richiede al Trulli un supplemento di collaborazione:

Pure, se ciò ad importunità non mi s'ascrive, io ho a pregar VS., sì come io hora la prego, voglia con la medesima sofferenza et cortesia fabricarmi la pari dimostratione dell'arterie; delle quali tutte la singolar celiaca per sì lungo giro lontana dall'aperta vena salvatella, vedesi contra l'opinion dello Spigelio non leggiermente pugnare. Ma via maggiore difficoltà parmi recare quest'altro argomento: che il sangue feccioso, dalla milza scosso et sottratto, montando su le parti superiori, debbia passare per lo sinistro seno del cuore, et $3^{0}$ che lui non contendano gli uscietti delle di lui membrane che fatte sono per rifiutare agli strani corpi l'entrata.

Il Trulli non aderì al nuovo desiderio del Severino, accampando a scusante, il 12 gennaio $1646^{127}$, una "gravissima lite in Ruota » ${ }^{128}$ e due viaggi «l'uno a Firenze (chiamato da quell'Altezza) et l'altro in Lombardia».

124 Seilo-phlebotome castigata, 151.

125 Seilo-phlebotome castigata, 152.

126 Lettera richiamata dalla nota 119.

127 Ms. Lancis. 11, c. $234^{\mathrm{r}}(=359)$. E' scritta da un amanuense e firmata dal Trulli, diversamente da tutte le altre lettere del Trulli da noi studiate, che sono invece olografe.

128 Decisiones Srae Rotae Romanae coram R.P.D.bon.mem. Coelio Bichio patritio senensi auditore celeberrimo, Roma 1671, 1, 183-186, 331-333, 367-368, 445-447 (Decisiones 98, 175, 193, 230). Debbo la notizia alla cortesia della Signora Bignami (cfr. nota 67) e di Mons. Charles Lefebvre, Uditore di Rota. 
Il Severino ritornò alla carica il $1^{\circ}$ novembre $1646^{129}$ e il 20 ottobre $1650^{130}$. Stralcio dalla prima di queste due lettere il seguente brano:

Exacta aestate, Phoca, trimestri telluris incolatu extinctus, mihi forte fortuna dissecandus obtigit; ex cuius inspecti amphibij observationibus autoschediasma confeci, styli dulcis, atque utilis usura mistum, synastomoseon amplissima discussione nobile, figuris illustre, theorematis, ac problematis in hoc genere inauditis praestabile, intra vigena typorum folia concludendum volumen; dicatum vero X. Europaeis maximis viris, sanguinem in corporis ambitu refluum professis; e quibus inclitus Licetus et M. Harveus, iam Coelo receptus, sunt praecipui, mox Castellus, et ipse, Trulli cum alijs assertoribus hoc numero dignis.

Quoniam autem memini de usu te Arteriarum in Harveiano dogmate serio dubitabili perplexum; hoc ego Problema non paucis exercui tuae, Trulli, praestantiae nuncupatum, quod mitto legendum, magis autem corrigendum, si quid peritiam tuam, aut Anatomicam, aut medicam offenderit fortasse; quod tempestive deleas, augeas, exascies, exornes.

Lo scritto del Severino annunciato in questa lettera apparve postumo nel 1659: Phoca illustratus: Anatomicum AYTOSXEDIASMA ${ }^{131}$ con la dedica «ad X. Viros ex tota Europa Clariss. Reflui in Orbem sanguinis assertores»: W. Harvey, G. Ent, N. Highmore, H. Conring, Th.Bartholin, W.Rolfinck, J.van Horne, V. F. Plemp, W. E. Scheffer, Pietro Castelli. Contrariamente alla promessa, il nome del Trulli non è stato incluso nel novero dei dieci. Il «problema Trullianum » ${ }^{132}$, la «Ambiguitas Joannis Trulli gravissima » ${ }^{133}$ è inveec fatta oggetto di diffusa trattazione ${ }^{134}$.

6. G.A.Borelli e M.Malpighi: iatromeccanica e scoperte strutturistiche (miocardio, capillari sanguigni). - Nella lettera già ricordata ${ }^{135}$ per la «cura, veramente maravigliosa, fatta dall'Eccl ${ }^{\text {mo }}$ Sig. Giovanni Trullo», Benedetto Castelli valuta al lume della fisica il comportamento volumetrico dell'intestino prolassato, e addita ai «moderni» anatomici il programma di ricerca iatromeccanico. Grazie ai loro progressi, essi hanno bensì «superato di gran lunga gli antichi filosofi», ma hanno anche preso coscienza «che ci

129 Ms. Lancis. 71 , c. 350 ; ms. Lancis. 74 , c. 275 (=290). Altra copia trovasi a c. 56 del ms. H 319 della Biblioteca medica dell'Università di Montpellier, donde fu trascritta da L. Amabile nella c. 172 del ms. XI. AA. 35 della Biblioteca Nazionale di Napoli.

130 Ms. Lancis. 74, c. 276.

131 E' parte integrante del volume Antiperipatias. Hoc est adversus Aristoteleos de respiratione piscium diatriba. De piscium in sicco viventibus. Commentarius in Theophrasti Eresii libellum huius argumenti. Phoca illustratus, scilicet anatome spectatus, et philosophico criterio examinatus. De radio turturis marini, eiusque vi, medicina, veneno, Neapoli, 1659.

132 Pp. 57 e 66.

133 P. 43.

134 Nel «Porisma de arteriarum apud Harveianos usu», pp. 43-66.

135 Richiamata dalla nota 99. 
restano ancora infiniti e maravigliosi artifizi e machine stupende da scoprirsi nella fabbrica dell'animale ${ }^{136}$, e del corpo umano in particolare, a segno che quello che fin ora è stato scoperto è una minima parte di quello che ci resta ancora da scoprire».

Siamo nel 1638: e proprio in quell'anno la scienza del moto ricevette i fondamentali Discorsi e dimostrazioni matematiche intorno a due nuove scienze attenenti alla mecanica e $i$ movimenti locali, in cui il Galilei non mancò di additare, in aggiunta ai precedenti ${ }^{137}$, qualche nuovo problema biofisico ${ }^{138}$. La sistematica applicazione della meccanica ai fenomeni del mondo vivente trovò il suo grande promotore in un allievo di B. Castelli, in Giovanni Alfonso Borelli (1608-79), che nel 1635 aveva lasciato Roma per ricoprire la cattedra matematica dell'Università di Messina. Già nel periodo Messinese ci sono attestati i suoi interessi iatromeccanici, che egli continuò a sviluppare fino al termine della sua vita, elaborando il De motu animalium, pubblicato nel 1680/1, all'indomani della sua morte. Un trentennio prima, nel 1649, era apparso a Cosenza il manifesto iatromeccanico del Borelli: il libro Delle cagioni delle febbri maligne della Sicilia negli anni 1647 e $1648^{139}$, che demolisce il tradizionale sistema quaternario a favore di una fisiologia e patologia atomistico-meccanica, comprensiva della statica del Santorio e della dottrina circolatoria:

... il principio, e il fonte di tutti i movimenti naturali, o mediatamente, o immediatamente è il Cuore, il quale, con la sua infaticabile agitatione, spigne con veloce corso il

136 Il corsivo è nostro!

137 Fondamentale è, in proposito, il brano de Il Saggiatore (1623) sulle qualità primarie e secondarie (Le opere di G. G., 6, 349). Si veda, ad es., L. Belloni, Suono e orecchio dal Galilei al Valsalva, Simposi Clinici 3 (1966) XXXIII-XLII. Sulla scoperta delle papille quali recettori sensoriali, è da vedere L. BeLloni, On the Discovery of the «Rete Malpighii» in Tongue and Skin, Archives of Dermatology 97 (1968) 101-109.

138 L.Belloni, La vessie natatoire des poissons selon Galilée et son école, et comme paradigme du mécanisme biologique, Colloque International sur l'Histoire de la Biologie marine, Banyuls-sur-Mer, 2-6 septembre 1963 (=Suppl. n. 19 a Vie et Milieu), Masson, Parigi 1965, 65-81; L. Belloni, Bionica del palombaro e del sommergibile dal Galilei al Borelli, Simposi Clinici 7 (1970) XvII-XxIv. Cfr. inoltre il lavoro qui citato alla nota 30 .

139 Una prima analisi di quest'opera ha dato materia alla mia conferenza Dal Borelli al Malpighi letta all'Università di Messina il 20 aprile 1967 per l'inaugurazione de $\mathrm{X}^{\circ}$ Congresso Nazionale di Patologia e stampata in Atti della Società Italiana di Patologia 10, 381-394, e in Simposi clinici 4 (1967) XVII-XXIv. La parte sostanziale della conferenza è inoltre riportata in M. MalPighi, Opere scelte a cura di L. Belloni, Utet, Torino 1967, 15-20. 
sangue fin alle estremità delle arterie; e quivi poi succhiato dall'estremità delle vene, si riconduce di nuovo al cuore, con giro veloce, e non interrotto (come ha dimostrato sensatamente l'Herveo $)^{140}$. hor in questo giro, occorre, che per l'impulso conferito al sangue dal dibattimento delle arterie, scappino via da i pori, e dalle estremità di esse innumerabili particelle, che nel sangue erano contenute; le quali con l'impeto ricevuto, insinuandosi ne gli spatij rimasti vacanti, dopo la traspiratione d'altre particelle, vengono con artificio maraviglioso a conservare quel flusso, e reflusso di parti, o quel movimento nel quale consiste la conservatione, e la vita dell'animale ${ }^{141}$.

La concezione atomistico-meccanica del Borelli trova applicazione anche in patologia. Febbre significa esagerazione del moto fisiologico:

... quel che noi chiamiamo moto febbrile, è una tale agitatione vehemente del Cuore, dalla quale per accidente, e come effetto ne seguita la fervenza del calore, perché gli spiriti, e il sangue caldi di natura, agitati, e spinti furiosamente per le arterie fin alla cute, e poi reiterando spesso con somma celerità il detto giro (conforme dimostrano l'esperienze d'Herveo $)^{142}$ vengono ad eccitare il detto riscaldamento ${ }^{143}$.

Le «febbri maligne» che danno materia al libro del Borelli, dipendono dalla inalazione e penetrazione in circolo di particelle tossiche:

la qualità velenosa, dal cuore per l'arterie, nelle vene, e poi nelle rimanenti parti comunicata, circolando insieme col sangue (conforme ha dimostrato l'Herveo ${ }^{144}$, huomo degno d'eterna gloria) può corrompere gli humori del corpo, e far sì, che le urine stravagantemente si alterino, e la febbre si aumenti, e si spanda furiosamente ${ }^{145}$.

Lasciata Messina, il Borelli iniziò nel 1656 l'insegnamento di matematica a Pisa, chiamato a quella Università dietro suggerimento del Michelini, che si era spontaneamente dimesso dalla cattedra. A Pisa il Borelli trovò l'ambiente ideale in cui sviluppare la sua iatromeccanica. In Toscana lo spirito di Galileo era sopravvissuto soprattutto nell'ambito della Corte granducale, e rifulse dal 1657 al 1667 nell'Accademia del Cimento.

Particolarmente importante fu l'incontro del Borelli con Marcello Malpighi (1628-94), chiamato proprio nel 1656 alla cattedra Pisana di medicina teorica. Il Malpighi era di un ventennio più giovane del Borelli: nel 1649 l'anno in cui apparve il libro Delle cagioni delle febbri maligne - egli si era deciso per lo studio della medicina, dietro consiglio di Francesco Natali (m. 1675), suo istitutore in filosofia peripatetica a Bologna. Il Malpighi divenne così allievo $\mathrm{d}$ Bartolomeo Massari (1603-55) e partecipò al gruppo di lavoro che questi fondò nel $1650^{146}$ in casa propria:

140 Il corsivo è nostro!

141 Borelli, Delle cagioni, 158-159.

142 Il corsivo è nostro!

146 H. B. Adelmann, M. Malpighi and the Evolution of Embryology, Ithaca 1966, 1, 103.
143 Borelli, Delle cagioni, 171-172.

144 Il corsivo è nostro!

145 Borelli, Delle cagioni, 128. 
Et quoniam tunc temporis sanguinis circulatio $^{147}$, aliaque nova experimenta anatomica famosa reddebantur; ideo Excellentissimus Massarius, curiositate motus, Chorum Anatomicum domi excitavit, electis novem suis auditoribus, inter quos locum habui. Huius Academiae institutum erat anatomicam lectionem stato tempore privatim habere, et frequentes sectiones moliri in hominum cadaveribus, et brutorum adhuc viventium corporibus ${ }^{148}$.

Nel 1653 in Malpighi conseguì, a Bologna, la laurea in filosofia e medicina. Nel 1656, come si è detto, fu chiamato a Pisa, dove, grazie all'influenza del Borelli, si staccò dalla filosofia peripatetica e abbracciò il metodo Galileiano : «Egli adunque si compiacque instradarmi nello studio della filosofia libera e Democritica, e da esso riconosco ciò che d'avanzamento io ho fatto filosofando. ${ }^{149} \mathrm{Il}$ Borelli aperse la propria casa a giovani ricercatori - come il Malpighi, Claudius Aubrius ${ }^{150}$ e, più tardi, Lorenzo Bellini (1643-1704) ${ }^{151}$ che, diversamente da lui, erano dotati di manualità anatomica, e li spronava alla ricerca degli «infiniti e maravigliosi artifizi e machine stupende da scoprirsi nella fabbrica dell'animale $»{ }^{152}$ alla ricerca, in altri termini, delle strutture anatomiche su cui fondare, meno idealmente di quanto aveva fatto il Cartesio, la propria concezione iatromeccanica. In testa ai reperti ottenuti in casa del Borelli figurano quelli sulla muscolarità del cuore e sulle fibre cardiache, descritti, fra l'altro ${ }^{153}$, nella Anatomia Italica ${ }^{154}$, dal Malpighi, il quale così conclude:

Ex his igitur probabiliter deduci potest, unum tantum fibrarum genus, loquendo de carne, adesse, quod scilicet a cordis basi exortum in eandem postremo desinat, harum

147 Il corsivo è nostro!

148 Dall'autobiografia del Malpighi in Opera posthuma, Londra 1697, p. 1.

149 Memorie di me Marcello Malpighi a i miei posteri fatte in villa l'anno 1689, conservate manoscritte alla Biblioteca Universitaria di Bologna e pubblicate nel fascicolo Nozze Boschi-Tomba, Bologna 1902.

150 L.Belloni, Dall'«occhiale» di Galileo all'anatomia microscopica di M.Malpighi: il «Testis examinatus» (Firenze, 1658) di Claudius Auberius, Rendiconti dell'Istituto Lombardo di Science e Lettere, Classe Scienze B 98 (1964) 205-226; L. Belloni, Die Entstehungsgeschichte der mikroskopischen Anatomie, Medizinische Monatsschrift 19 (1965) 113-122 e in R. Herrlinger e F. Kudlien, Frühe Anatomie. Von Mondino bis Malpighi. Eine Anthologie, Stoccarda 1967, 269-296.

Il Lorenese Claudius Aubrius, chiamato nel 1656 alla cattedra anatomica di Pisa, proveniva dall'Università di Padova, dove si era immatricolato presso la Natio Germanica Artistarum il 2 maggio 1652 e si era laureato in filosofia e medicina il 9 febbraio 1654. Cfr. p. 361 del lavoro di Weigle qui citato alla nota 41.

151 F. Grondona, L'esercitazione anatomica di Lorenzo Bellini sulla struttura e funzione dei reni, Physis 5 (1963) 423-463.

152 Dalla lettera di B. Castelli richiamata dalla nota 136. 
tamen aliquas magis vel minus spirales fieri, ex quibus conflatur cor, musculus verus et perpetui motus auctor ${ }^{155}$. Huius autem structurae exactior historia, mecanicusque usus ab ipso $^{156}$ in desiderato de motu animalium libro ${ }^{157}$ habebitur.

Identica conclusione era stata pubblicata nel 1664 da Niels Stensen (Steno, 1638-86) ${ }^{158}$ : «... in corde nihil desiderari, quod musculo datum, nec quod musculo denegatum, in corde inveniri : non erit cor amplius sui generis substantia, adeoque nec ... ignis, calidi innati ... sedes.» ${ }^{159} \mathrm{Il}$ cuore, infatti, ha la stessa temperatura di altri grandi visceri - come dimostrarono ricerche termometriche compiute dal Borelli durante il periodo Pisano (1656-67) e non può quindi essere considerato «focus praecipuus animalis, qui ob excessivum ardorem refrigerari et ventilari debeat».160

Ma l'apparato del moto circolare del sangue è ancora gravemente lacunoso dal punto di vista anatomico. Se il cuore funge da centro propulsore, che immette il sangue nei condotti arteriosi e lo riceve di ritorno tramite $i$ condotti venosi, devono essere dimostrati i finissimi condotti che stabiliscono l'anastomosi fra arterie e vene.

153 Alludo alle testimonianze postume dei due autori, purtroppo in reciproca polemica: G.A. Borelli, De motu animalium, Roma 1681, 2, 90, propos. XXXviI; M. MalPIGHI, Opera posthuma, Londra 1697, 2. La partecipazione rispettiva dei due autori corrisponde verosimilmente a quella indicata dal Malpighi nell'Anatomia Italica, di cui diremo alla nota 154: «Circa cordis carnem non est transeundum id, quod Praeeunte praeclarissimo Jo: Alfonso Borellio Pisis in elixato corde observavi ...»

${ }_{154}$ E' una storia dell'anatomia che il Malpighi aveva iniziato a scrivere nel periodo Messinese (1662/6), tosto interrompendola per l'amichevole premura del Borelli stesso e di Carlo Fracassati, preoccupati che questa attività letteraria potesse compromettere l'attività sperimentale del Malpighi. Il testo trovasi nel ms. 2085/II, pp. 362-401 della Biblioteca Universitaria di Bologna e fu edito da G. Atri, Notizie edite e inedite della vita e delle opere di Marcello Malpighi e di Lorenzo Bellini, Bologna 1847, 54-72.

155 Il corsivo è nostro!

156 Ossia dal Borelli.

157 Il De motu animalium del Borelli, che, come si è detto, apparve postumo nel 1680/1, è già preannunciato nello stesso De pulmonibus (Bologna, 1661) del Malpighi. Cfr. M. MalPighi, Opere scelte a cura di L.Belloni, Utet, Torino, 1967, 99.

158 N.Steno, De musculis et glandulis observationum specimen, Hafniae, 1664; ristampato in Opera philosophica edited by V.Maar, Copenhagen 1910, 1, 181.

159 I corsivi sono nostri!

160 G. A. Borelli, De motu animalium, Roma 1681, 2, 189 (cap. viII, propos. Xcvi: «Respirationem institutam non esse ad refrigerium et ventilationem flammae et caloris cordis»). La termometria cardiaca ritorna a p. 445 (cap. XXI, propos. G CXXI: «Non a foco, vel fermento in corde existente, febris accenditur»). 
Nel 1659, insofferente del clima Pisano, il Malpighi rientrò a Bologna: e nel 1660 - applicando il microscopio alla ricerca delle minute macchine dell'organismo animale - compì le grandi scoperte che tosto annunciò al suo maestro Borelli nelle due lettere De pulmonibus (Bologna 1661) ${ }^{161}$ : la struttura alveolare del parenchima polmonare e la rete dei vasellini capillari che stabilisce l'anastomosi artero-venosa ${ }^{162}$.

L'apparato meccanico postulato dalla dottrina circolatoria è finalmente completo nell'intero giro. La scoperta dei capillari - che presuppone l'impiego dell'ingrandimento ottico, elevato a strumento di scienza dal Galilei fu compiuta da un ricercatore, come il Malpighi, che scientificamente dal Galilei discende, tramite il Borelli, emanazione del gruppo Galileiano di Roma, così precocemente curioso per la dottrina della circolazione del sangue. E' una feconda generazione scientifica, che si continua negli allievi del Malpighi - come G.M.Lancisi, I.F.Albertini, A.M. Valsalva e, tramite questi, G.B.Morgagni - che alla dottrina della circolazione impartiranno sviluppi anche nell'ambito della patologia.

Il presente saggio di raccolta, e reciproca connessione, di testimonianze relative all'accettazione e all'assimilazione della dottrina circolatoria da parte della Scuola Galileiana, trova la sua giustificazione sia negli importanti sviluppi che quella dottrina ricevette da questa stessa Scuola, sia nell'ambito più generale del problema dei primordi della iatromeccanica. L'argomento è di tale rilievo, che mi propongo di svilupparlo, con nuovo apporto di documenti, lungo la traccia abbozzata nella presente nota.

Oltre alla Signora Bignami e a Mons. Lefebvre, già ricordati alle note 67 e 128, desidero ringraziare quanti altri hanno agevolato le mie ricerche, e in particolare: Miss J.M. Backhouse, Assistant Keeper, Department of Manuscripts, British Museum, London; il Dr. Marcello Del Piazzo, direttore dell'Archivio di Stato, Roma; Mme. Annie Fabre-Aubrespy, Conservateur p.i., Bibliothèque, Section de Médecine, Université de Montpellier; il Dr. Massimo Fittipaldi, direttore della Biblioteca Nazionale, Napoli; la Dr.Luigia Risoldi, direttore, e il Cav. Luigi D'Aurizio della Biblioteca Universitaria, Bologna; la Dr. Lucia Rossetti dell'Istituto per la Storia dell'Università di Padova; la Dr. G. Scano, direttore dell'Archivio Storico Capitolino, Biblioteca Romana; l'Avv. Luciano Tului, direttore, e i suoi collaboratori della Biblioteca Lancisiana, Roma; il Dr. Nello Vian, Segretario della Biblioteca Apostolica Vaticana, Roma. Rivolgo anche un deferente pensiero alla memoria del Bibliotecario Lancisiano Pietro De Angelis.

161 M.MalPighi, Opere scelte a cura di L.Belloni, Utet, Torino 1967, 69-99.

162 L. BeLloni, The discovery of blood capillaries (De pulmonibus by M.Malpighi, 1661), The Journal of Cardiovascular Surgery 3 (1962) 464-471; I capillari sanguigni nelle tavole del Malpighi, Physis 5 (1963) 70-77. 